\title{
Cooperative Charging Management for Electric Vehicles via Mobile Edge Computation
}

Yuanjian Zhang ${ }^{1}$, Chong Guo ${ }^{1}$, Chao Sun², Zheng Chen ${ }^{3,4^{*}}$, Guang $\mathrm{Li}^{4}$ and Yonggang $\mathrm{Liu}^{5 *}$

${ }^{1}$ State Key Laboratory of Automotive Dynamic Simulation and Control, College of Automotive Engineering, Jilin University, Changchun, 130022, China

${ }^{2}$ National Engineering Laboratory for Electric Vehicles, School of Mechanical Engineering, Beijing Institute of Technology, Beijing, 100081, China

${ }^{3}$ Faculty of Transportation Engineering, Kunming University of Science and Technology, Kunming, 650500, China

${ }^{4}$ School of Engineering and Materials Science, Queen Mary University of London, London, E1 4NS, UK

${ }^{5}$ State Key Laboratory of Mechanical Transmission \& School of Automotive Engineering, Chongqing University, Chongqing, 400044, China

Corresponding Authors: Zheng Chen (chen@kust.edu.cn) and Yonggang Liu (andylyg@umich.edu)

\begin{abstract}
Unplanned charging supervision of electric vehicles may deteriorate their penetration in alleviating pollution and reducing the driving efficiency, and proper management is critical to reduce charging waiting time and efficiently design driving behaviours from spots to charging stations. Motivated by this, a novel bifunctional charging management strategy in virtue of the mobile edge computation based framework is proposed in this study to effectively book the charging piles with less waiting time and meanwhile achieve better energy efficiency during charging booking. First, a novel charging booking algorithm is developed to determine the most suitable charging station and optimally plan the shortest route to the preferred charging station. Second, a driving behaviour optimization method is designed to plan the efficient velocity profile of the trip to the selected station under the constrained time calculated by the charging booking algorithm. The simulation analysis validates that the proposed bi-functional management strategy can reasonably book suitable charging stations and efficiently reduce energy consumption in the charging booking process, highlighting its anticipated preferable performance.
\end{abstract}

Key words: Charging management strategy (CMS), charging booking algorithm (CBA), driving behaviour optimization (DBO), mobile edge computation (MEC), electric vehicles (EVs)

\section{INTRODUCTION}

Nowadays, severe social conflicts among development, environment and natural resources expedites technology evolution [1-3]. Academia and industries have spared no effect to dramatically mitigate these passive concerns by means of transportation electrification $[4,5]$. Electric vehicles (EVs), as one of ideal solutions that completely avoid greenhouse gas emission, have attracted substantial attention [6]. Recent prominent progress in charging infrastructure construction accelerates market penetration of EVs [7]. Despite the achievement in development of charging facilities, great challenges still exist in charging management of EVs [8]. Improper charging management may adversely affect daily use and acceptance of EVs in aggravating the range anxiety and triggering indirectly other social issues, such as traffic congestion and travel disruption. Indeed, charging management has become one of vital problems in EV promotion that deserves to be properly tackled. 
The EV charging management, generally, can be divided into two categories: fixed charging scheduling (FCS) for EVs when parking at homes, and dynamic charging administration (DCA) for on-the-move EVs [911]. Since the FCS mode is relatively direct and easy to be tailored, in this study, we focus mainly on developing optimal strategies for DCA of on-the-move EVs. Presently, a variety of progress has been made for the development of on-the-move charging management and the main task can be divided into the following three steps: appropriate charging planning, management of CSs to grid and path planning from spots to CSs. The charging planning mainly accounts for selecting a suitable CS with less waiting time and optimally allocating the charging schedule to reduce cost and grid load. Ref. [12] proposes an efficient coordinated management strategy for on-the-move EVs to reduce the charging waiting time. Actually, the designed publish/subscribe framework enables reduction of the charging time to a large extent. A case study in Helsinki manifests the preferable performance of raised strategy. Ref. [13] reveals a charging scheduling algorithm to minimize the charging cost and charging load. The daily charging demand profile is predicted and incorporated into the charging appointment of each EV. Management from CSs to the power grid is mainly focused on rationally allocating load to CSs from power grid based on the charging requirement while keeping the grid stable. Ref. [14] introduces an instant smart load management solution by optimizing the maximum sensitivity selection, whereby the EV charging can be scheduled more flexibly to minimize the grid overload and promote its stability and reliability. Ref. [15] successfully minimizes the load at CSs by heuristic based manners and genetic algorithm (GA). Based on the proposed method, the CS can determine whether they can meet the charging request under the constraint of current grid load. Currently, path plan for EV charging from spots to CSs has been actively investigated. By means of effective path plan, efficient routes from spots to CSs can be generated along with optimization of different targets, such as energy consumption and travelling time. Actually, path plan can be regarded as a dynamic optimization problem and undoubtedly can be effectively solved by typical optimization algorithms, such as dynamic programming (DP) and its extensions including stochastic DP (SDP) and approximate DP (ADP) [16]. In [17], the charging booking problem of individual EV owners is considered, and the shortest path on an extended transportation graph is searched. A so-called social planner is employed to determine the optimal route with less travel time and energy consumption.

Existing solutions for charging management claim to accomplish charging scheduling in on-board vehicle control units (VCUs) or global controllers (GCs) located in cloud servers; however, despite of VCUs or GCs, the burdensome computation load incurred by complex algorithms weakens their real-time application potential. The rapid development of communication technologies [18], artificial intelligence [19] and remote sensing technologies [20] promote the research with respect of charging booking management to a new stage. In this context, the emerged mobile edge computation (MEC) accelerates further optimization in terms of charging management. Ref. [21] applies the MEC to integrally resolve charging management and manifests the favourable improvements. In addition to satisfactory performance in general charging management, more exploration and/or exploitation is encouraged with respect to MEC based charging management for wider implementation scenarios. In most applications, charging booking is necessary when electric energy/power is limited. Moreover, how to arrive at CSs with minimum energy consumption should also be incorporated when 
scheduling charging booking. Even though the path plan of charging management can provide efficient routes, it is not comprehensive, and particularly driving behaviours can also affect energy consumption. If driving behaviour optimization (DBO) can be integrated into charging management, it would be further beneficial for energy saving and efficiency improvement for charging management. To the best of the authors' knowledge, there is no released research and publications in terms of the combined optimizations.

DBO has been one of mainstream directions in the development of driving assistant systems (DAS) for both EVs [22] and autonomous vehicles [23]. DAS is capable of generating optimal suggestions on driving manoeuvres to achieve preeminent energy economy and driving safety. The optimal instructions of energysaving and driving-securing DAS are usually interpreted from the inclusively planned solution supplied by DBO, which are optimal velocity profiles in most cases [24-29]. On this basis, DAS can efficiently guide drivers to manipulate EVs, or control autonomous vehicles, to follow the planned routes and keep safe distances from front vehicles with adaptive velocity. In [26], a computationally efficient multi-layered control strategy is proposed for velocity optimization, thereby contributing to performance improvement of ecodriving. The strategy divides the driving task into several operation modes, and model predictive control (MPC) is applied to enable fast regulation. Ref. [27] comes up with an ecological driving system based on estimation of distribution algorithm (EDA) and MPC. The reference velocity profiles considering energy efficiency is generated by the EDA, and then the MPC is employed to track the reference profiles, thereby adaptively following front vehicles. The current studies on DBO, briefly summarized, employ global methods to generate the optimal or near-optimal velocity profiles with preferable trade-off of computation and storage intensity and then try to follow them by the enabled methods. No doubt, the inherent time-consuming characteristic of global optimization methods deteriorates possibility of real-time implementation. To enable faster generation of optimal reference velocity profiles, Ref. [30] introduces iterative DP (IDP) to realize ecodriving control of EVs. IDP, compared to conventional DP, can achieve satisfactory accuracy of optimal solution with less computation duration through an adaptive objective function, whereby the reference velocity trajectories corresponding to given travel time can be iteratively obtained. Given this reason, IDP can be a qualified candidate for EV charging management. As far as we know, the application of IDP in a more specific scenario that is rarely reported in the literature.

According to the literature review, most of the raised CMSs focus on the pre-booking process in the charging management. However, the after-booking process is seldom considered in previous studies that is of the vital importance to energy saving. The after-booking process mainly accounts for planning shortest or efficient paths to the chosen CSs and optimizing velocity profiles in the trips to the chosen CSs. Motivated by this, a novel CMS for EVs is herein proposed based on a service oriented MEC framework. The CBA in the brand new strategy books the charging request after comprehensively considering multiple constraints and plans the shortest route to the chosen CSs. Then, the IDP is subsequently executed to achieve the DBO, thereby minimizing the energy consumption from spot to the reserved CS. The main contributions of this study can be attributed to the following two main aspects: 
1) A novel CBA is developed in the raised CMS to reduce the charging waiting time exhaustively. The most suitable CS is picked up by comprehensively considering traffic conditions, workload of CSs and vehicle status. After charging is booked, the CBA also takes charge of planning the shortest route to the chosen CS from current spot by the A-star algorithm.

2) The DBO is firstly incorporated into the CMS. The IDP optimizes velocity profiles from spots to CSs with the pre-set travel time estimated by the CBA. The optimal velocity profiles are tracked by applying the MPC algorithm in DASs of EVs, thereby generating detailed optimal manoeuvre suggestions. Through the DBO based on the IDP and MPC algorithm, the electricity consumption of EVs in the trips of spots to the chosen CSs is remarkably saved with the strictly constrained travel time that is given according to the charging available time, prompting energy economy of EVs in charging processes.

The remainder of this paper is organized as follows. The EV is modelled in Section II. Section III presents the newly developed CMS. Section IV discusses the simulation results and comparatively validates the performance of the raised strategy, followed by the main conclusions drawn in Section V.

\section{FoundATION OF THE CMS: VeHICLE MODELING}

In this section, the EV model is built to help development of the CMS, and it mainly includes the vehicle dynamic model and powertrain model, of which the latter consists of the motor model and battery model. The vehicle dynamic model and powertrain model are briefly described in the following separate parts.

\subsection{Vehicle Dynamic Model}

The preferred EV is driven by a single motor placed in the front axle. The torque between wheels and motor is directly transmitted via the final gear. The main parameters of studied EV are listed in Table 1 . The dynamic relationship between the motor torque and vehicle dynamic can be expressed as:

$$
\begin{aligned}
\dot{v}(k) & =\frac{1}{m}\left(\frac{g_{f r} N_{f r}}{r_{w}} T_{m o t}(k)-\frac{1}{2} \rho C_{d} A_{d} v(k)^{2}-m g f \cos \theta(k)-m g \sin \theta(k)-\frac{T_{b r k}}{r_{w}}\right) \\
& =\frac{1}{m}\left(P_{1} T_{m o t}(k)-P_{2} v(k)^{2}-P_{3}-\frac{T_{b r k}}{r_{w}}\right)
\end{aligned}
$$

where $P_{1}=g_{f r} N_{f r} / r_{w}, P_{2}=\frac{1}{2} \rho C_{d} A_{d}$, and $P_{3}=m g f \cos \theta(k)+m g \sin \theta(k)$. In (1), $T_{m o t}$ denotes the torque provided by the motor; $m, g_{f r}, N_{f r}$ and $r_{w}$ denotes the vehicle mass, final gear efficiency, final gear ratio, and wheel radius, respectively; $\rho, C_{d}, A_{d}$ and $v$ expresses the air density, aerodynamic drag factor, frontal area, and vehicle speed, respectively; $g, f$ and $\theta$ represents the gravity acceleration, rolling resistant factor, and road gradient, respectively; and $T_{\text {brk }}$ indicates the mechanical braking torque. The relationship among the vehicle acceleration, velocity and tractive torque is explained in (1). Obviously, it is in time domain. However, for ease of CMS development, the relationship can be transferred into the distance domain, as:

$$
v(k+1)=v(k)+\dot{v}(k) \frac{2 \Delta s}{v(k)+v(k+1)}
$$


where $\Delta s$ is the calculation step in distance, $k$ and $k+1$ denote the geographic location at current and next steps. By combining (2) and (1), we can attain:

$$
v(k+1)^{2}=\left(1-\frac{2 \Delta s}{m} P_{2}\right) v(k)^{2}+\frac{2 \Delta s}{m} P_{1} T_{m o t}(k)-\frac{2 \Delta s}{m} \frac{T_{b r k}}{r_{w}}-\frac{2 \Delta s}{m} P_{3}
$$

Furthermore, equation (3) can be reformulated into:

$$
v(k+1)^{2}=A v(k)^{2}+B T_{m o t}(k)-C T_{b r k}-D
$$

where $A=1-\frac{\Delta s}{m} \rho C_{d} A_{d}, B=\frac{2 \Delta s g_{f r} N_{f r}}{m r_{w}}, C=\frac{2 \Delta s}{m r_{w}}$, and $D=\frac{2 \Delta s}{m}(m g f \cos \theta(k)+m g \sin \theta(k))$.

Table 1 Main Parameters of EV

\begin{tabular}{lll}
\hline \multirow{2}{*}{ Item } & Variable & Values \\
\hline \multirow{3}{*}{ Vehicle } & Vehicle Mass & $1552 \mathrm{~kg}$ \\
\cline { 2 - 3 } & Wheel Radius & $0.307 \mathrm{~cm}$ \\
\cline { 2 - 3 } & aerodynamic drag coefficient & 0.28 \\
\hline \multirow{2}{*}{ Battery } & Type & Lithium-ion battery \\
\cline { 2 - 3 } & Capacity & $60 \mathrm{Ah}$ \\
\cline { 2 - 3 } & Nominal Voltage & $330 \mathrm{~V}$ \\
\hline \multirow{2}{*}{ Motor } & Maximum Power & $90 \mathrm{~kW}$ \\
\cline { 2 - 3 } & Maximum Torque & $300 \mathrm{Nm}$ \\
\hline \multirow{2}{*}{ Performance } & Max Speed & $160 \mathrm{~km} / \mathrm{h}$ \\
\cline { 2 - 3 } & Max Travel Mileage & $170 \mathrm{~km}$ \\
\hline
\end{tabular}

\subsection{Vehicle Powertrain Model}

\subsubsection{Motor Model}

In this study, a permanent magnet synchronous motor is equipped in the vehicle. Considering the DBO target in this paper, the electric motor dynamic behaviours are not taken into account for simplifying the optimization. The static performance of electric motor can be described by a stationary model. The electric motor can operate as either a tractive motor or a generator, and the relationship between the motor torque and power can be described, as:

$$
P_{\text {mot }}= \begin{cases}\frac{T_{m o t} \omega_{e m}}{\eta_{\text {mot }}} & T_{e m}>0 \\ T_{\text {mot }} \omega_{e m} \eta_{g e n} & T_{e m} \leq 0\end{cases}
$$

where $\omega_{e m}$ is the angular speed of electric motor; $P_{\text {mot }}$ is the power of electric motor; and $\eta_{\text {mot }}$ and $\eta_{\text {gen }}$ are the motor efficiency in tractive mode and generator mode, respectively. The motor efficiency can be located from the look-up table calibrated through the benchmark test. To accelerate the computation speed in DBO, the motor efficiency look-up table is approximated by a multi-order polynomial function. Considering the fitting accuracy and calculation complexity in control algorithm, we prefer a 5th-order polynomial function, which is acquired by the curve fitting of the 3D look-up table, as: 


$$
P_{m o t}=\left\{\begin{array}{l}
\eta_{m o t}=a+b m(x, y)+c m^{2}(x, y)+d m^{3}(x, y)+e m^{4}(x, y)+f m^{5}(x, y) \\
m(x, y)=a_{10} x+a_{01} y+a_{11} x y \\
m^{2}(x, y)=a_{20} x^{2}+a_{02} y^{2}+a_{21} x^{2} y+a_{12} x y^{2}+a_{22} x^{2} y^{2} \\
m^{3}(x, y)=a_{30} x^{3}+a_{03} y^{3}+a_{31} x^{3} y+a_{13} x y^{3}+a_{32} x^{3} y^{2}+a_{23} x^{2} y^{3} \\
m^{4}(x, y)=a_{40} x^{4}+a_{04} y^{4}+a_{41} x^{4} y+a_{14} x y^{4} \\
m^{5}(x, y)=a_{50} x^{5}+a_{05} y^{5}
\end{array}\right.
$$

where $x$ is the motor speed $\omega_{e m}$, and $y$ denotes the motor torque $T_{m o t}$. The parameters in (6) are estimated offline by the particle filter method introduced in [31]. The relationship among the motor torque, generator torque and vehicle tractive torque can be expressed as:

$$
T_{\text {mot }}= \begin{cases}\frac{T_{\text {trac }}}{\eta_{\text {mot }} N_{f r}} & T_{\text {trac }}>0 \\ T_{\text {mot }} N_{\text {fr }} \eta_{\text {gen }} & T_{\text {trac }} \leq 0\end{cases}
$$

where $T_{\text {trac }}$ is the vehicle tractive torque.

\subsubsection{Battery Model}

Lithium-ion batteries are a complex system that can be influenced by temperature and aging effect. The designed CMS, however, considers single charging booking process every time. Consequently, it is fair to assume there exists limited impact on charging booking performance influenced by temperature and aging degradation of battery each time. In other words, the designed CMS books charging piles with instantly unvaried battery degradation every time. The main target of this paper is to present the integrated CMS properly, which can definitely be outstretched to consider more constraints such as battery degradation in the future. Therefore, considering features of CMS, work target and modelling complexity, the temperature influence and aging effect are neglected, and a simple but effective equivalent circuit model is employed to characterize the battery's electric performance. The model consists of an internal resistance and an open circuit voltage source connected in series topology, whereupon the battery current can be calculated, as:

$$
I_{\text {batt }}=\frac{V_{\text {batt }}-\sqrt{V_{\text {batt }}^{2}-4 r_{\text {batt }} P_{\text {batt }}}}{2 r_{\text {batt }}}
$$

where $I_{\text {batt }}$ and $V_{\text {batt }}$ denote the battery current and open circuit voltage; $r_{\text {batt }}$ and $P_{\text {batt }}$ is the battery inner resistant and power, respectively. The battery state of charge (SOC) can be calculated by:

$$
S \dot{O} C=-\frac{V_{\text {batt }}-\sqrt{V_{\text {batt }}^{2}-4 r_{\text {batt }} P_{\text {batt }}}}{2 r_{\text {batt }} Q_{\text {batt }}}
$$

where SOC is the battery SOC, and $Q_{b a t t}$ is the battery capacity. The detailed description of the derivation of (8) and (9) is provided in our previous work [32]. In EV charging process, the charging battery power $P_{\text {batt_cha }}$ is calculated based on grid power, efficiencies of charging pile, battery, and can be expressed as:

$$
P_{\text {batt_cha }}=P_{\text {grid }} \eta_{\text {cha_p }} \eta_{\text {cha_m }} \eta_{\text {batt_cha }}
$$


where $P_{\text {grid }}$ means the power at the grid side, $\eta_{c h a_{-} p}$ denotes the efficiency of charging pile, $\eta_{\text {cha_m }}$ represents the efficiency of the on-board charging machine, and $\eta_{\text {batt_cha }}$ is the battery charging efficiency. The efficiency of charging piles $\eta_{\text {cha }}$, the on-board charging machine $\eta_{\text {cha_m }}$ and the battery charging efficiency $\eta_{\text {batt_cha }}$ is set to $95 \%$, $88 \%$ and $92 \%$, respectively.

\section{THE DEVELOPMENT OF NOVEL CHARGING STRATEGY}

\subsection{The Service-Oriented MEC Based Framework}

Commercialization of the fifth-generation (5G) communication technologies [33] and emergence of MEC promote the superior-performance charging management [34, 35]. The network edge owns powerful computing capacity [35], and some control process can be executed at network edge instead of the vehicle. In this case, the vehicle needs to offload the full or part control task to network edge via wireless network. In the wireless network, the millimeter wave with ultra-wide bandwidth is used. The implementation of the millimeter wave is on the basis of the sparse radio frequency (SRF) chain antenna structure at sides of vehicle and the base stations (BSs) of network with less hardware cost and power consumption [36]. In this context, a service-oriented MEC based framework, as shown in Fig. 1, is constructed which is well suited to charging management particularly.

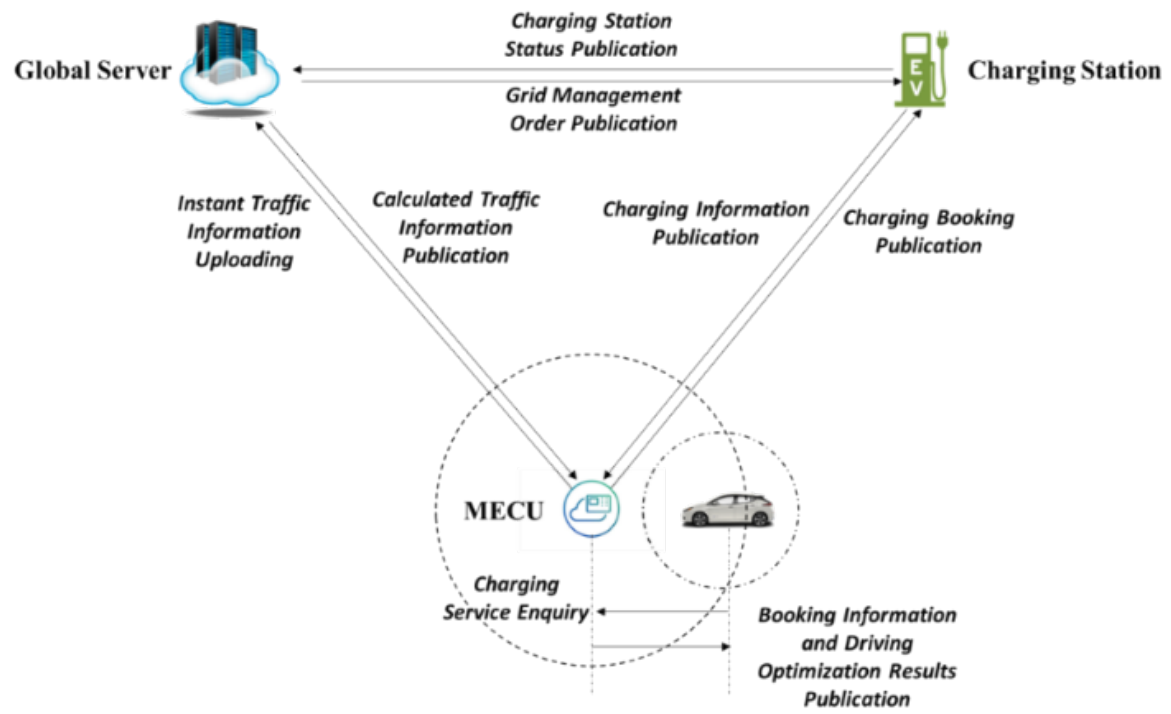

Fig. 1. Illustration on the service oriented MEC framework.

The MEC based framework, actually, is a service-oriented paradigm where the stakeholders including EVs and charging stations (CSs) subscribe the valuable information published in the framework. Drivers of EVs are permitted to book charging piles via the optimal management conducted in mobile edge computing units (MECUs).

3.2 Development of the Novel CMS 
The novel CMS is developed under the MEC based framework to accomplish charging management in MECUs. The brief charging management procedure based on the developed framework can be described as follows.

Step 1: EV publishes the charging request to the network. The MECU that holds the smallest distance to the EV subscribes the charging requirement. Then, the corresponding MECU starts the charging booking. During this process, the inputs and outputs of MECUs, CSs and global server (GS) can be:

\section{GS}

Input: the instant traffic information from MECUs and the state information of CSs that includes number of available CSs, numbers of available charging piles in each CS, geographic location of CSs, instant traffic flow velocities, etc.

Output: the grid adjustment order to CSs and the processed traffic information to MECUs includes data of road network, road information (such as speed limits), etc.

\section{CSs}

Input: the adjustment order on grid from GS and the charging booking order from MECU.

Output: the state information to MECUs and GS, which includes available charging piles, geographic location of CSs, etc.

\section{MECUs}

Input: the state information of CSs and the processed traffic information.

Output: the charging booking order, the shortest path to the chosen CS and the optimized velocity profile.

Step 2: Once the MECU that is nearest to EV subscribes the charging request, it picks up and books the candidate CS with less waiting time by the CBA based on the shared CS state information. The CBA also finds the shortest path to the chosen CS by referring the route network information and instant traffic information from GS.

Step 3: After booking the charging service in certain CSs, DBO is performed in MECUs. The DBO is a two-stage process that is implemented in both MECUs and on-board VCU. In the first stage of DBO, MECUs will generate the optimal velocity profile of the trip to the chosen CS through the cooperation with GS. According to the planned travel route by CBA, GS will assign the optimization work to certain MECUs that is beside the given travel route. It is worth noting that each MECU just generates the optimal velocity profile for next route segment based on the collected instant traffic information, which can prompt the ability of proposed CMS in real-time application. Moreover, the velocity profile of the first route segment of the travel to the chosen CS is also optimized by the MECU for the first route segment.

Step 4: With the optimized velocity profile transmitted from MECUs, the on-board VCU generates the optimal suggestion for driving manoeuvre in the second stage of DBO.

The proposed CMS fully takes advantage of the raised MEC framework to achieve the reasonable and remarkable charging management through the cooperative interaction among GS, MECUs and EV. The flowchart of the designed CMS is shown in Fig. 2 (a). During the charging management, the charging mode selection, charging reservation, path plan, travel time estimation and DBO are performed in sequence. Among 
all the crucial tasks, the CBA accounts for the charging mode selection, charging reservation, path plan and travel time estimation. The DBO method generates optimal velocity profiles for the energy-efficient driving. The optimal velocity profiles generated by DBO are sent to vehicles via V2I communication.

\subsubsection{CBA Development in CMS}

Some researchers have performed the study on the CBA and raised some solutions by different framework [37] with the consideration of various factors such as power system technical constraints [38]. In this paper, we focused on the integrated control process in CMS which includes CBA and DBO. Therefore, the influence on charging pile available time from various factors like power system technical constraints is neglected. The optimization task of CBA is to book the most appropriate CSs and plan the reasonable route from the spots to the CSs. In the designed CMS, there exist two charge booking modes. The two charge booking modes are determined according to user behaviours or mandatory order such as the forced charge when the left electric energy in vehicles is low. Some users prefer to charge EVs immediately due to the serious range anxiety, while it is acceptable for other users to charge EVs after finishing the current journey. These two modes are also suitable for cases that drivers subjectively want to charge EVs in which energy in batteries is barely consumed. Despite the clear division in charge mode, the two modes can be mutually switched under some conditions. The charge mode can be switched from 1 to 2 or inversely from 2 to 1 if drivers change their mind. Once the charge mode switches to 1 form 2 , the route will be planned again to find the most efficient route to the original travel destination. Even though drivers insist to choose mode 2, the charge mode will switch to 1 if the left electric energy cannot sustain the current trip.

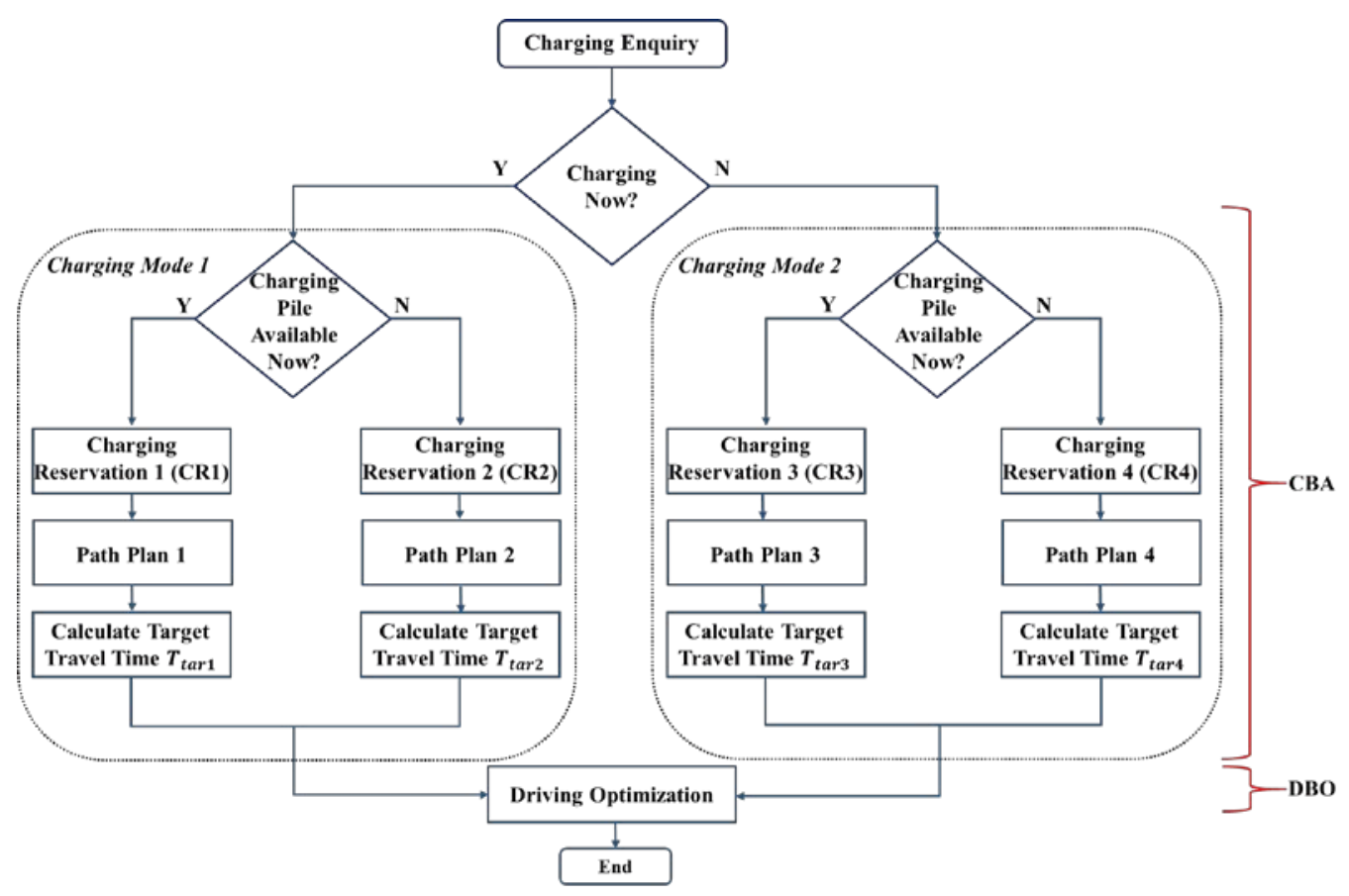

(a) 


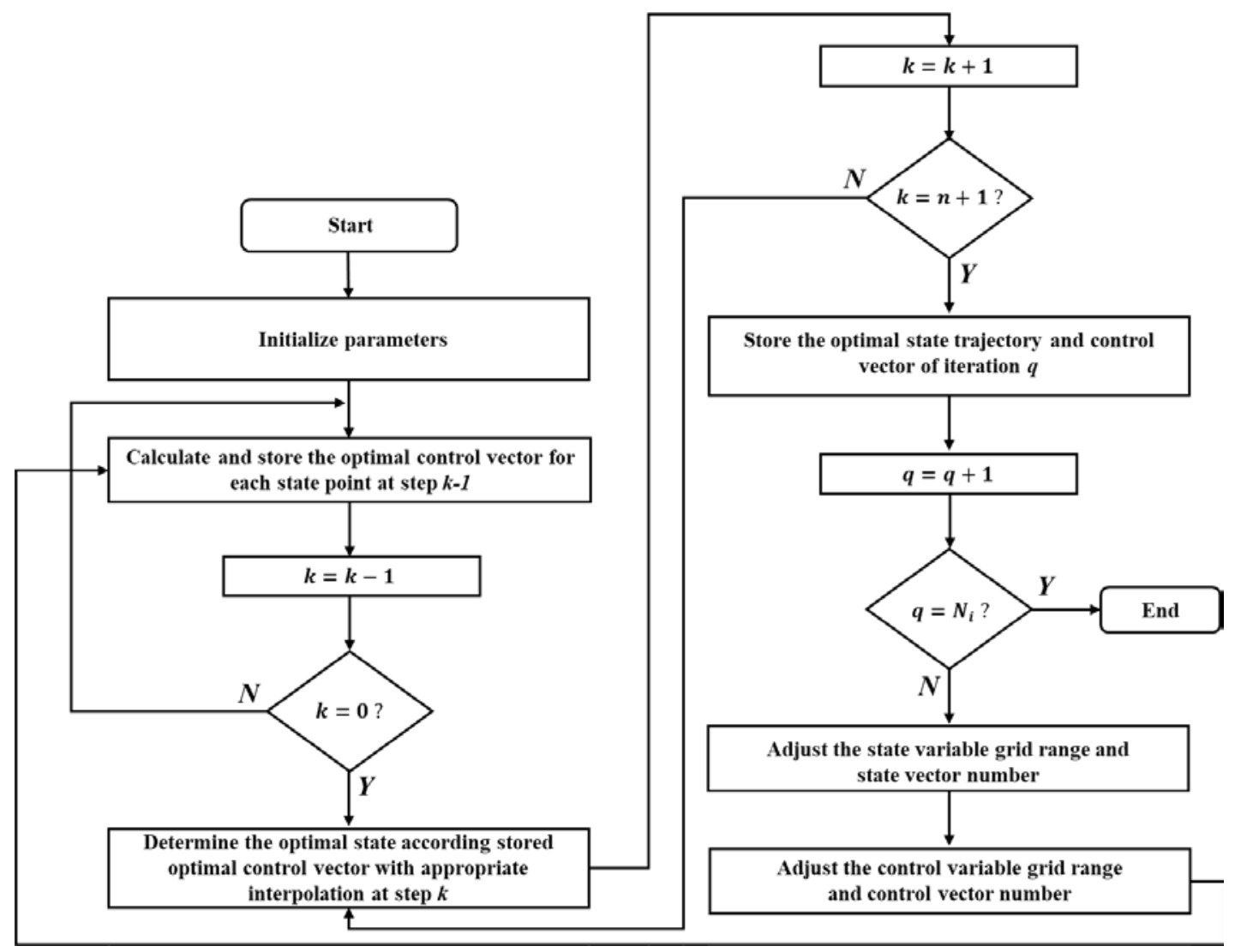

(b)

Fig. 2. Illustration on method implementation. (a) Flowchart of CMS. (b) Illustration of IDP implementation.

Mode 1: When the battery SOC is lower than the pre-set threshold or drivers demand battery charging, EV needs to connect with CSs, and the charging booking is reserved immediately.

Mode 2: Charging is expected after the current journey. The vehicle is scheduled to be charged after finishing the current trip, and the corresponding charge booking is initiated.

The difference in the two mode is the timing that charging is required. Charging is required immediately in mode 1 while the charging is required after current journey is completed in mode 2 . In mode 1 , charging request makes the predefined travel waived. However, the charging process will not sacrifice current travel in mode 2. In these two modes, the CBA is only activated after the charging order is given. The vehicles will approach to the end of travel positions along the planned routes with the minimum route length in both modes 1 and 2. On the travel to the end of travel positions, driving behaviours are optimized by the DBO to improve the energy consumption economy. When designing the logic in CS booking, the principle is to achieve minimum waiting time when EV arrives at CS. If the CS can provide available charging piles immediately or before EV arrives at CS, it will be chosen as the candidate CS in charging booking. Among the candidate CSs, the CS with the maximum charging piles available time will be specified as the booked CS. The pseudocodes of the developed CBA in two charging modes are presented in respectively Tables 2 and 3, where the ramp mode means that vehicle runs with a very low speed, which is around 5 to $10 \mathrm{~km} / \mathrm{h}$. In addition, the DBO in Tables 2 and 3 will be introduced in following section. 
Table 2 Pseudocode of CBA in charging mode 1

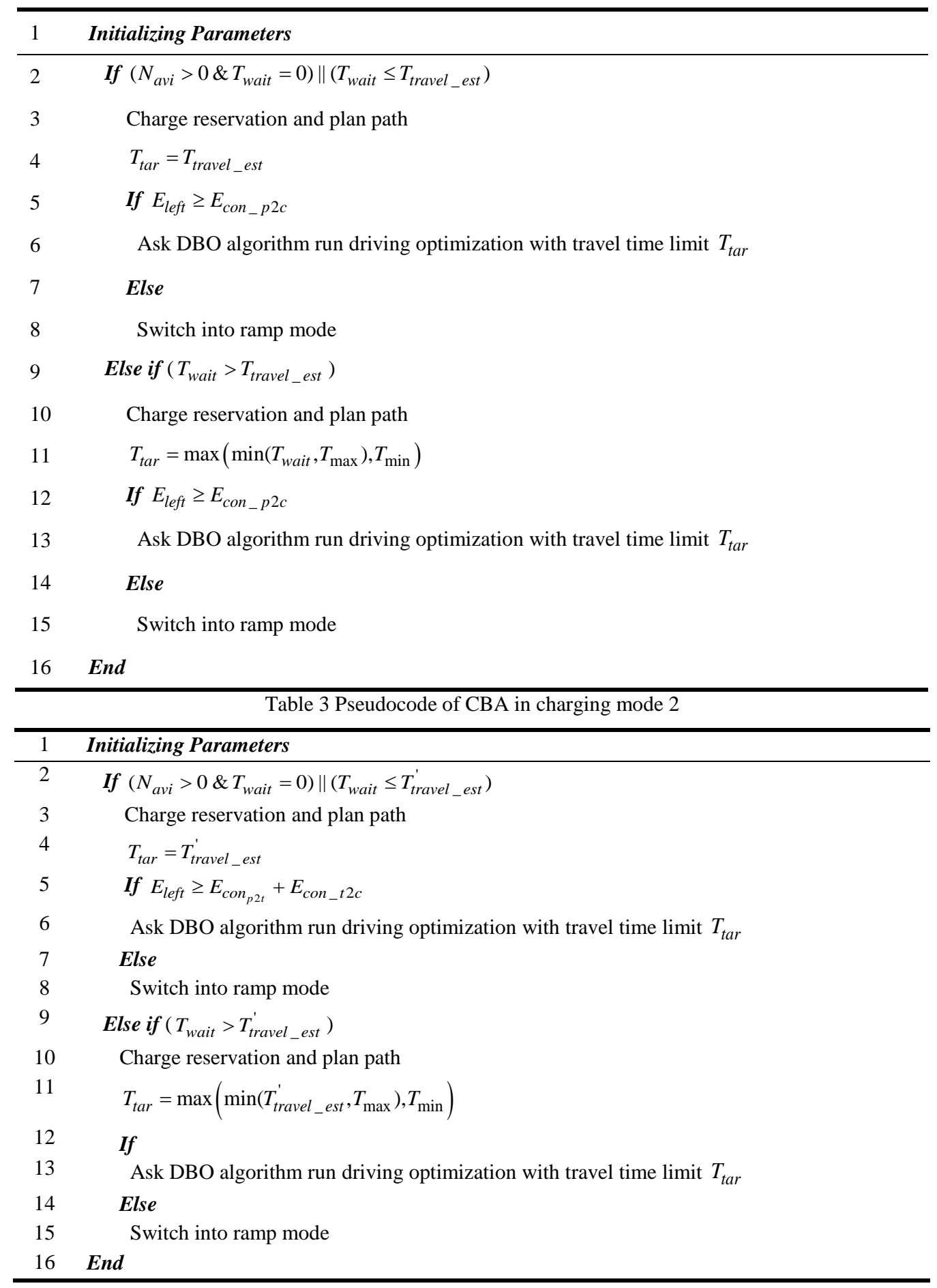

After the charging pile in certain CS is booked, the path plan is performed to find the shortest route from current spot to the chosen CS. By this manner, the travel time from current spot to the chosen CS can be optimized to be minimum. In this study, the widely accepted A-star algorithm is applied to plan the shortest path, and it employs the heuristic function to evaluate cost from the candidate node to the destination node in the road network [39]. In the MEC based charging booking, MECU that is closest to EV will receive the processed traffic information such as road network data and instant traffic flow velocities from GS. Based on the given road network data and other data, the A-star algorithm will search the most appropriate route segment 
combination with minimum cost under the given search direction. In this study, the cost discussed here is the route length. The function to assess the cost can be expressed as:

$$
f(x)=l(x)+h(x)
$$

where $f$ denotes the evaluated cost from the candidate node to the destination, $l$ is the optimal cost from the starting node to the current node, and $h$ means the heuristically estimated cost from current node to the destination. The pseudocode of A-star algorithm is presented in Table 4.

Table 4 Pseudocode of A-star algorithm

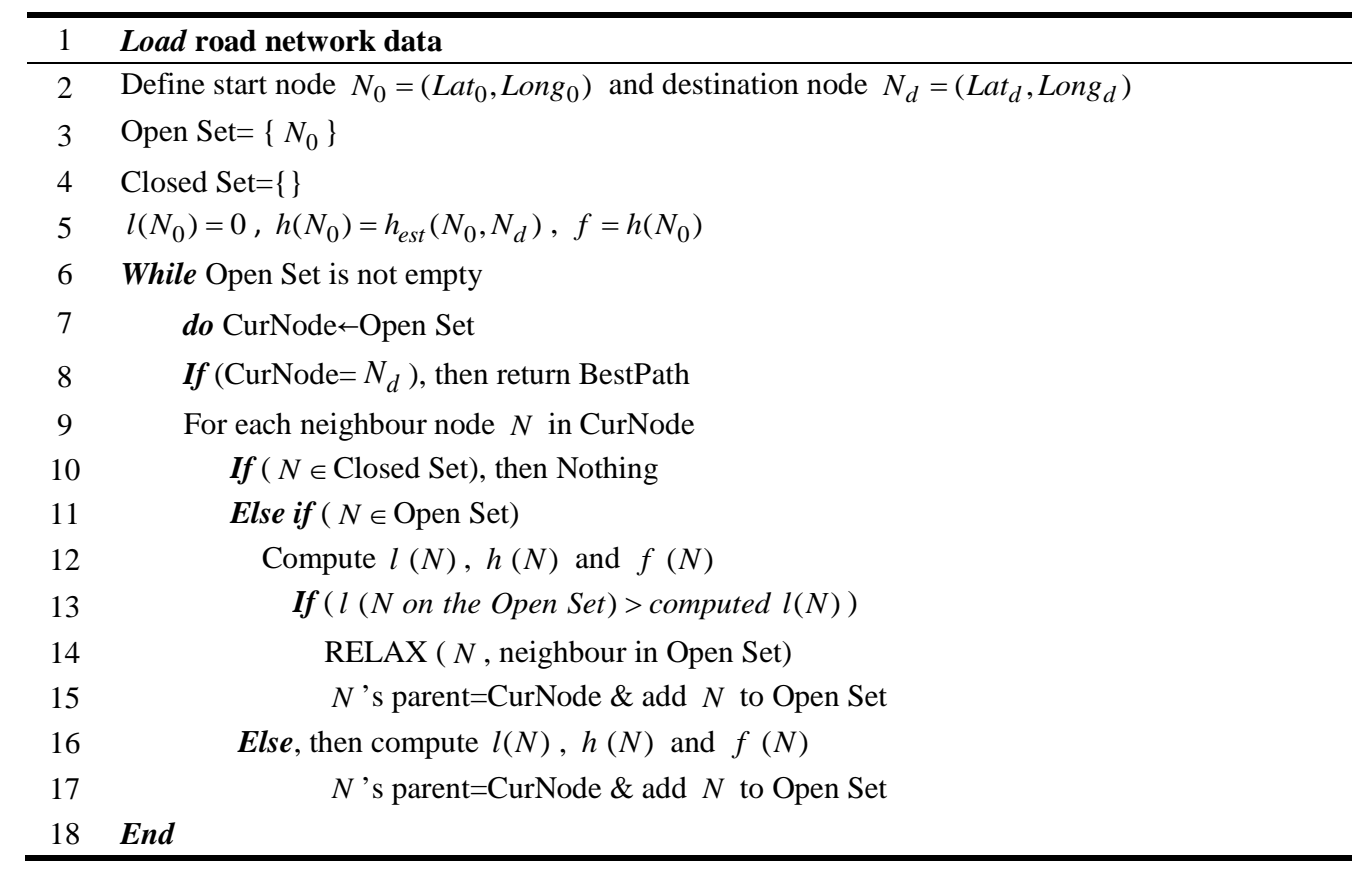

As can be found in Tables 2 and 3, the average travel times $T_{\text {travel_est }}$ and $T_{\text {travel_est }}^{\prime}$ on the planned route are estimated based on the traffic flow in route segments. The detailed process of calculating $T_{\text {travel_est }}$ and $T_{\text {travel_est }}^{\prime}$ can be found in our previous work [40]. With the shared traffic flow velocities and estimated average travel times, the required energy $E_{c o n_{-} p 2 c}, E_{c o n_{p 2 t}}$ and $E_{c o n_{-} t 2 c}$ for traveling from spots to CSs in Tables 3 and 4 can be calculated by:

$$
E=\int_{0}^{T_{\text {tar }}} \frac{v}{\eta_{t}}\left(\frac{G f \cos \theta}{3600}+\frac{G \sin \theta}{3600}+\frac{C_{d} A_{d} v^{2}}{76140}+\frac{\xi m a}{3600}\right) d t
$$

where $G, \eta_{t}, \xi$ and $a$ denotes the gravity, transmission efficiency, correction coefficient of rotation mass, and acceleration, respectively.

\subsubsection{DBO Method in CMS}

The essence of DBO is to acquire optimal velocity profiles for the energy-efficiency driving on the chosen route. The implementation of designed DBO method is a two-step process: generation and tracking of the optimal velocity profiles. The generation of the optimal velocity profile is a global optimization process in MECUs, avoiding much on-board computation pressure. In each MECU, the optimal velocity profile for next route segment will be generated by IDP. MECU that is in charge of the first route segment will also generate 
the velocity profile for the current route segment by parallel computing. After the on-board VCU receives the optimal velocity profile, the MPC algorithm will be implemented to track the optimal velocity profile, thereby generating the optimal suggestion for driving manoeuvres.

\section{A. IDP Method Implemented in Velocity Profile Optimization}

The velocity profile optimization is essentially a nonlinear optimal control problem that is defined to minimize the cost function within a finite horizon, as:

$$
J=H(x(t), u(t), t)+\int_{0}^{t_{f}} L(x(t), u(t), t) d t
$$

where $x$ and $u$ denotes the state and control inputs, respectively; $t$ is the time step; $t_{f}$ is the time at final step, $H$ is the terminal cost; and $L$ is the stage cost. Additionally, the problem is subject to the given inequality constraints:

$$
\left\{\begin{array}{l}
x_{\min } \leq x(t) \leq x_{\max } \\
u_{\min } \leq u(t) \leq u_{\max } \\
g(x(t), u(t), t) \leq 0
\end{array}\right.
$$

The stage cost in IDP can be formulated as:

$$
L_{k}\left(x_{k}^{i}, u_{k}^{j}\right)=L_{k}\left(v_{k}^{i}, T_{k}^{j}\right)=\omega_{t} \cdot \frac{2 \cdot P_{\text {batt }}\left(v_{k}^{i}, T_{k}^{j}\right) \cdot \Delta s}{v(k)+v(k+1)}+T_{b r k}\left(v_{k}^{i}, T_{k}^{j}\right)
$$

where $\Delta s$ is the step length, and $\omega_{t}$ is the weight ratio. As a numerical method, IDP can achieve the approximate optimal solution, compared to DP, while consuming much less computation sources. The implementation process of IDP is depicted in Fig. 2 (b), and the comparison between DP and IDP is illustrated in Fig. 3 (a). As can be seen, the boundaries of IDP is obviously narrower than those in DP, and consequently less discrete state and control variables and acceleration of the calculation speed can be anticipated.

For the velocity profile optimization in CMS, the optimization targets include the energy-efficient velocity profile and pre-estimated travel time from spot to the chosen CS. To fulfill the optimization targets, IDP is implemented with a tunable weight ratio that restricts the travel time and is adaptively adjusted between two iterations. In this study, $\omega_{t}$ in (15) can be tuned innovatively by:

$$
\omega_{t}(q+1)=\omega_{t}(q)+\operatorname{sign}\left(T_{\text {real }}(q)-T_{\text {target }}(q)\right) \cdot \delta e^{\left(T_{\text {real }}(q)-T_{\text {target }}(q)\right)^{2}}
$$

where $q$ is the iteration time, $T_{\text {real }}$ is the real travel time, and $T_{\text {target }}$ is the target travel time, which can be expressed as:

$$
T_{\text {real }}=\int_{0}^{N_{s}} \frac{2 \Delta s}{v(k)+v(k+1)} d \tau
$$

where $N_{s}$ is the number of calculation steps. In (16), the current weight ratio is $\omega_{t}(q)$, and the weight ratio of next step $\omega_{t}(q+1)$ can be iteratively calculated by comparing the difference between $T_{\text {real }}$ and $T_{\text {target }}$. For IDP implementation, the functions to adjust the grid sizes of state and control variable can be redesigned, as: 


$$
\left\{\begin{array}{l}
x_{k_{-} \min }^{q+1}=\max \left(x_{k_{-} \min }^{q}, x_{k_{o p t}}^{q}-\alpha \cdot\left(x_{k_{-} \max }^{q}-x_{k_{-} \min }^{q}\right)\right) \\
x_{k_{-} \max }^{q+1}=\min \left(x_{k_{-} \max }^{q}, x_{k_{\text {opt }}}^{q}+\alpha \cdot\left(x_{k_{-} \text {max }}^{q}-x_{k_{-} \min }^{q}\right)\right) \\
u_{k_{-} \min }^{q+1}=\max \left(u_{k_{-} \min }^{q}, u_{k_{\text {opt }}}^{q}-\beta \cdot\left(u_{k_{-} \max }^{q}-u_{k_{-} \min }^{q}\right)\right) \\
u_{k_{-} \max }^{q+1}=\min \left(u_{k_{-} \max }^{q}, u_{k_{\text {opt }}}^{q}+\beta \cdot\left(u_{k_{-} \max }^{q}-u_{k_{-} \min }^{q}\right)\right) \\
n_{u_{-} k}^{q+1}=\max \left(n_{u_{-} k}^{\min }, n_{u_{-} k}^{\max } \cdot \frac{x_{k_{-} \max }^{q+1}-x_{k_{-} \min }^{q+1}}{x_{k_{-} \max }^{q}-x_{k_{-} \min }^{q}}\right) \\
n_{x_{-} k}^{q+1}=\max \left(n_{x_{-} k}^{\min }, n_{x_{-} k}^{\max } \cdot \frac{u_{k_{-} \max }^{q+1}-u_{k_{-} \min }^{q+1}}{u_{k_{-} \max }^{q}-u_{k_{-} \min }^{q}}\right)
\end{array}\right.
$$

where $x_{k_{-} \max }^{q+1}, x_{k_{-} \min }^{q+1}, u_{k_{-} \max }^{q+1}$ and $u_{k_{-} \min }^{q+1}$ denote the upper and lower boundaries of state variables and control variables at step $k$ in iteration $q+1 . x_{k_{\text {opt }}}^{q}$ and $u_{k_{\text {opt }}}^{q}$ are the optimal state and control variable at step $k$ in iteration $q . u_{k_{-} \max }^{q}$ and $u_{k_{-} \min }^{q}$ are the upper and lower boundary of control variables at step $k$ in iteration $q$. $n_{u_{-} k}^{q+1}$ and $n_{x_{-} k}^{q+1}$ are the numbers of discrete state and control variables at step $k$ in iteration $q+1 . n_{u_{-} k}^{\max }, n_{u_{-} k}^{\min }$ , $n_{x_{-} k}^{\max }$ and $n_{x_{-} k}^{\min }$ represent the maximum and minimum values of the discrete control and state variables at step $k . \alpha$ and $\beta$ are the contracted ratios. During the optimization process, some inequality constraints of the powertrain components are also taken into account, as:

$$
\left\{\begin{array}{l}
S O C_{\text {min }} \leq S O C \leq S O C_{\text {max }} \\
P_{\text {batt_min }} \leq P_{\text {batt }} \leq P_{\text {batt_max }} \\
T_{\text {mot_min }} \leq T_{\text {mot }} \leq T_{\text {mot_max }} \\
\omega_{\text {em_min }} \leq \omega_{\text {em }} \leq \omega_{\text {em_max }}
\end{array}\right.
$$

where the subscripts $\min$ and $\max$ denote the minimum value and maximum value of each variable, respectively. Fig. 3 (b) to (d) illustrate the implementation process of IDP in a given route with the permitted maximum speed. As clearly illustrated in Fig. 3 (b), the IDP algorithm searches the optimal solutions in a narrowed field. Hence, the velocity profiles in each iteration solved by IDP can adaptively vary. Fig. 3 (c) and (d) present the adjustment of constraints of state and control variables. As the iteration goes on, the boundries of state and control variables are gradually shrinked, thus accelerating the optimization process. The reduced boundries also manifest that the solution approaches to the optimal result gradually. 


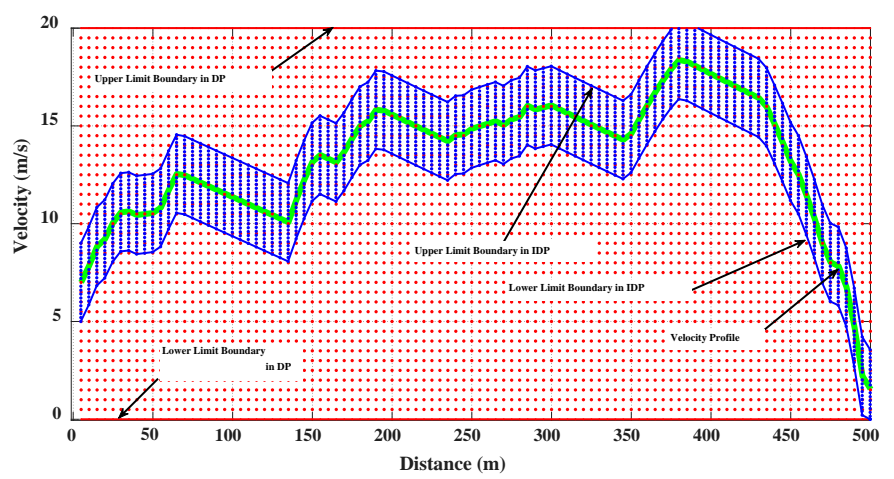

(a)

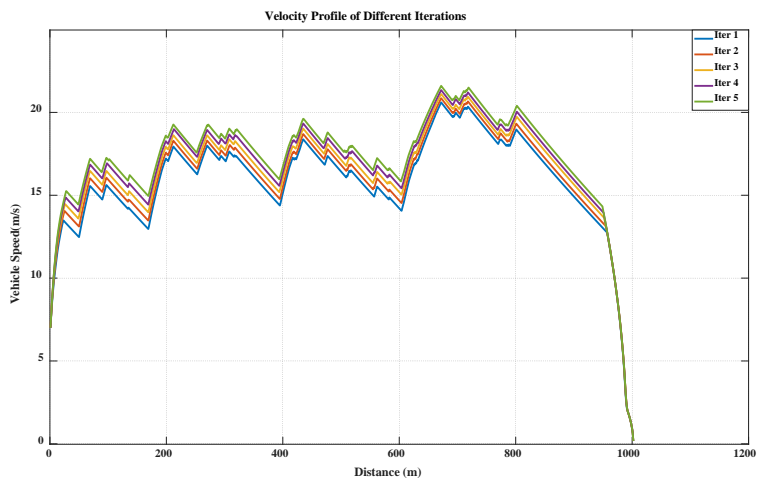

(b)

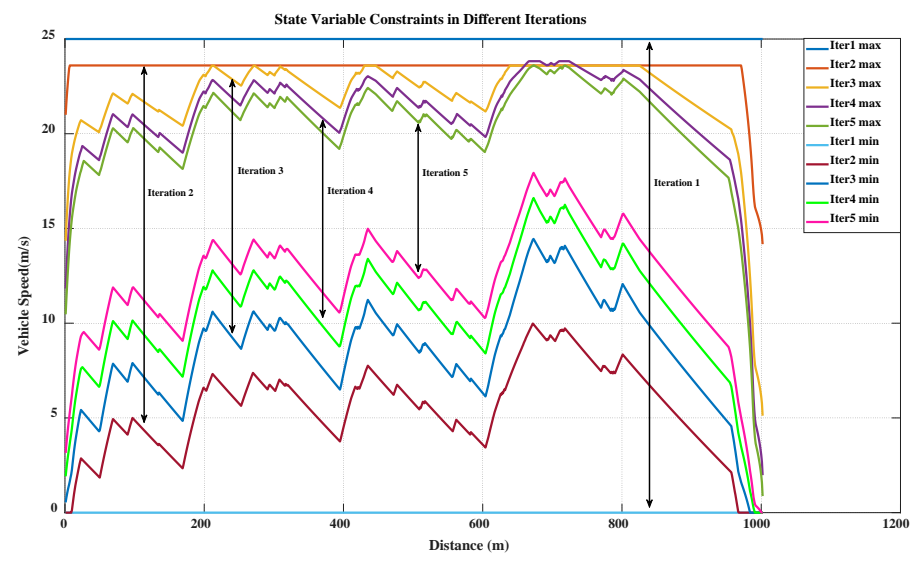

(c)

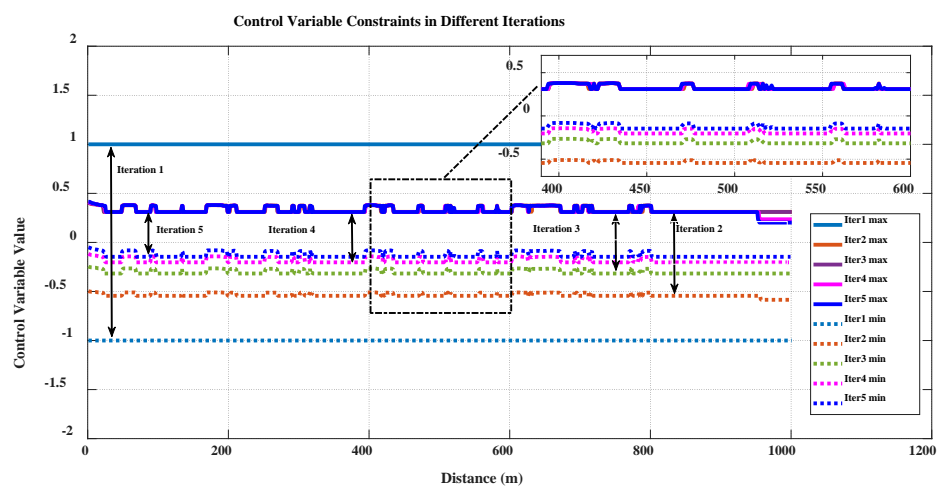

(d)

Fig. 3. Illustration of IDP features. (a) Comparison in execution process between DDP and IDP. (b) Velocity profile of each iteration by IDP. (c) State variable constraint in each iteration by IDP. (d) Control variable constraint in each iteration by IDP.

\section{B. MPC in Optimal Reference Tracking}

The obtained optimal velocity profile can be tracked by a nonlinear MPC to realize energy-efficient driving in conventional EVs or autonomous EVs. The calculation step in the nonlinear MPC is $2 \mathrm{~m}$, and the horizon length for the receding optimization is set to $10 \mathrm{~m}$. In the optimal tracking MPC problem, the optimization target can be formulated, as:

$$
U^{*}(k)=\arg \min \sum_{i=1}^{n_{p}}\left\|y(k+i)-y^{*}(k)\right\|_{Q}^{2}+\|\Delta u(k+1)\|_{R}^{2}
$$


subject to:

$$
\left\{\begin{array}{l}
x(k+1)=A x(k)+B u(k) \\
u(k)=u(k-1)+\Delta u(k) \\
y(k)=C x(k)+D u(k) \\
u_{\text {min }} \leq u(k) \leq u_{\text {max }} \\
x_{\text {min }} \leq x(k) \leq x_{\text {max }} \\
y_{\text {min }} \leq y(k) \leq y_{\text {max }}
\end{array}\right.
$$

where $y$ is the control output, $Q$ and $R$ are the weighting matrixes, and $n_{p}$ is the prediction length. The optimal tracking problem in (20) can be reformulated as:

$$
\min J=\min \left(\hat{y}^{T} Q_{1} \hat{y}+\hat{x}^{T} Q_{2} \hat{x}+\hat{u}^{T} R \hat{u}\right)
$$

where $Q_{1}$ and $Q_{2}$ denote the penalty matrixes. The problem mentioned in (25) can be converted to a $\mathrm{QP}$ problem, as:

$$
\min J=\min \left(\frac{1}{2} \hat{u}^{T}\left(\Psi^{T} Q_{2} \Psi+R\right) \hat{u}+x(k)^{T}\left(\Phi^{T} Q_{2} \Psi\right) \hat{u}\right)=\min \left(\frac{1}{2} \hat{u}^{T} S \hat{u}+x(k)^{T} W \hat{u}\right)
$$

subject to:

$$
P \hat{u} \leq M_{1}+M_{2} x(k)
$$

where $S, W, P, M_{1}$ and $M_{2}$ are the constant matrices. In this case, the required motor torque and mechanical braking torque are the control variables, and the cost function in MPC can be formulated as:

$$
J_{c}(k)=\sum_{i=1}^{n_{p}}\left(v(i \mid k)-v_{\text {opt }}(i \mid k)\right)^{2}
$$

subject to:

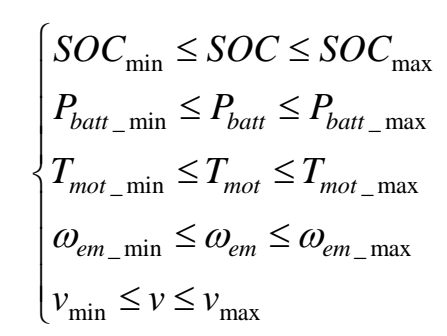

where $v_{\max }$ and $v_{\min }$ denotes the maximum and minimum velocity limits on the route segments, respectively.

\section{SIMULATION AND EVALUATION}

To validate performance of the proposed CMS, we present a comprehensive simulation evaluation in this section. The active roles of CMS in CS reservation and DBO are emphatically investigated. To analyze the CS reservation by CBA, a demonstrated EV charging system is constructed. In the built charging system, six CSs are included with the provision of different charging power. The numbers of available piles in each CS are assigned, and the preset battery SOC threshold under which EVs require immediate charging is 0.2 . For the evaluation of DBO, the referred optimal velocity profiles generated by IDP and tracked by MPC are both carefully evaluated. In the simulation, we assume that each MECU takes charge of one route segment with the 
length of 500 m. We resort to a workstation with an Intel Xeon E3-1270 @ 3.4 GHz and 32 Gigabytes memory to perform the simulation. Note that the data for building the charging system are obtained from the opening sources in [41, 42].

\subsection{Analysis Performance of the Novel CMS in Charging Booking}

In the CBA evaluation, two alternative methods are considered as benchmarks, including the daily travel habit based method (DTH) and the charging reservation strategy with minimum travel time to CSs (MTD). The novel raised charging booking algorithm in the evaluation is expressed as NCBA. The chosen CSs in simulation are located in the urban zone, London, and the detailed position is shown in Fig. 4. To better evaluate the performance of CBA in the novel CMS, the chosen CSs are all publicly available. The related parameters of CSs are listed in Table 5. Fig. 5 (a) and (b) illustrate the time comparison solved by three different methods which are respectively based on MEC and non-MEC framework. S2CS in Fig. 4 means from the spot to $\mathrm{CS}$, and the total time before charging denotes the charging waiting time which also includes the travel time from the spot to CS.

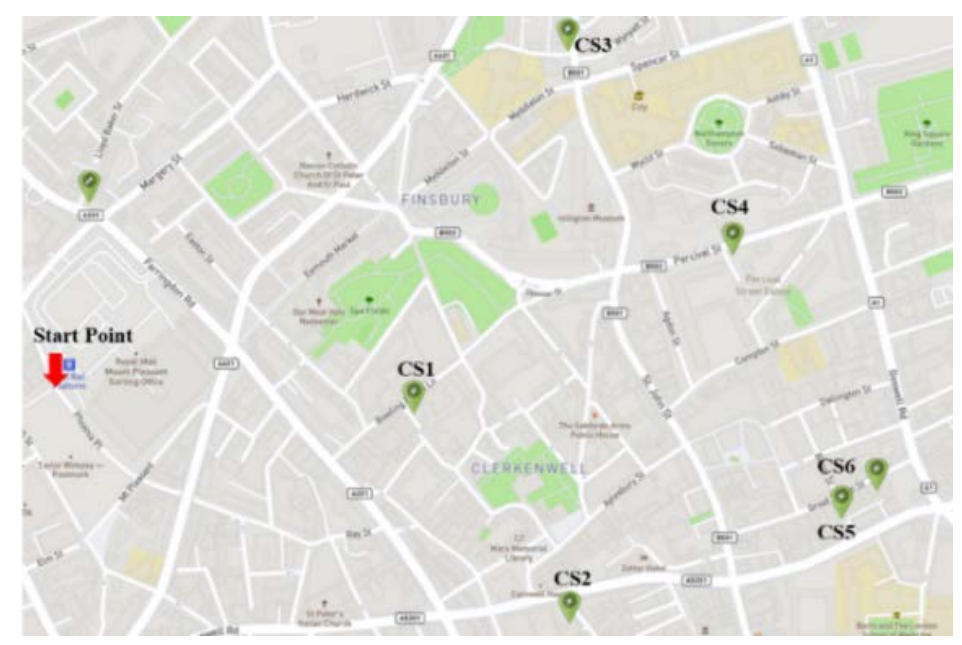

Fig. 4. Locations of CSs on the map.

In Fig. 5 (a) and (b), the S2CS travel time comparison aims to analyze the performance of route selection in the proposed CBA, while the comparison of total time before charging provides clear understanding of the general ability of proposed CBA. The comparison of total time before charging is built on the condition that different charging booking algorithms select the same CS. As illustrated in Fig. 5 (a) and (b), the travel duration by NCBA to each CS from the starting point is all less than that by DTH. The travel time by NCBA can be saved by up to $13.2 \%$ with the MEC based framework and 6.1\% with the non-MEC based framework. In other words, the CBA in the novel CMS can find the shortest path from spot to CS, compared to that by DTH, which contributes to savings of the charging waiting time and energy consumption. Meanwhile, the total time before charging by the NCBA in each CS is shorter than that solved by MTD. The total time before charging is reduced by $26.3 \%$ with the MEC based framework and $16.3 \%$ with the non-MEC based framework. The comparison results manifest that the proposed NCBA with the MEC based framework can select the most appropriate CS with the consideration of both travel time from spot to CS and charging pile available time, highlighting better performance than existing methods. 
Table 5 Parameters of charging piles in CSs

\begin{tabular}{llll}
\hline Charging Station & Charging Piles Number & Charging Power (kW) & Charging Voltage (V) \\
\hline CS1 & 6 & $4 / 7$ & 230 \\
CS2 & 4 & $4 / 7$ & 230 \\
CS3 & 4 & $4 / 7$ & 230 \\
CS4 & 4 & $4 / 7$ & 230 \\
CS5 & 2 & $4 / 7$ & 230 \\
CS6 & 4 & $4 / 7$ & 230 \\
\hline
\end{tabular}
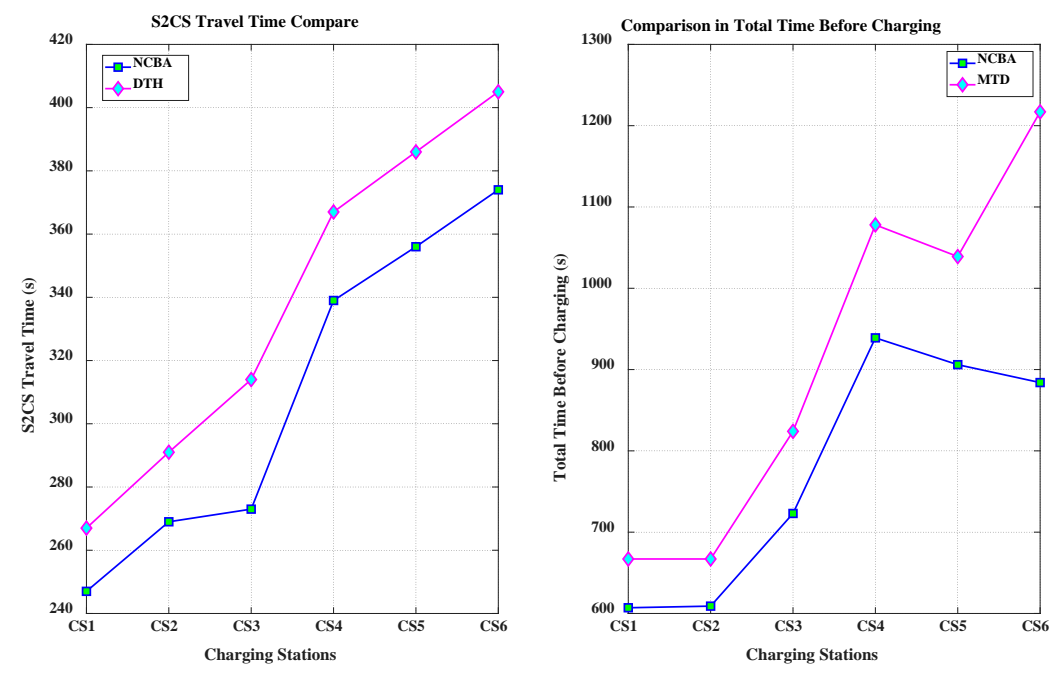

(a)
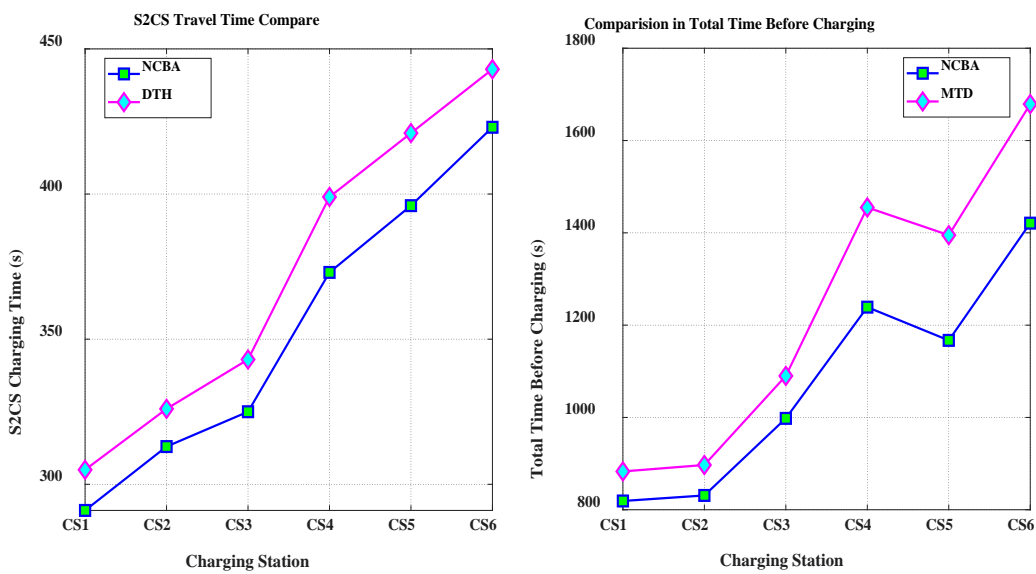

(b)

Fig. 5 Comparison among different charging booking algorithms. (a) Comparison among different charging booking algorithms based on MEC based framework. (b) Comparison among different charging booking algorithms based on non-MEC based framework.

The path planning in NCBA contributes to the apparent reduction in travel time to each CS and total time before charging. Some deeper evaluation on the path planning algorithm is also conducted, including the Astar method and Dijkstra algorithm. As well known, the Dijkstra algorithm exhaustively searches the solution under given constraints. Table 6 show the simulation results by the A-star method and Dijkstra algorithm. The results indicate that the A-start method can complete the route planning faster, compared with that by the Dijkstra algorithm. The computing time of the A-star method is only $2.5 \%$ of that by Dijkstra. The Dijkstra algorithm can search the most optimal route with the shortest length in exhaustive search. However, the A-star 
achieves better trade-off between computing time and planning results and is therefore more suitable in the novel CMS.

Table 6 Simulation results by A-star and Dijsktra

\begin{tabular}{lll}
\hline Method & Computing Time (s) & Route Length $(\mathrm{m})$ \\
\hline A-star & 1.731 & 1343 \\
Dijsktra & 46.416 & 1321 \\
\hline
\end{tabular}

\subsection{Evaluation of the DBO in Novel CMS}

\subsubsection{Investigation on Reference Velocity Profiles Generation}

To validate the computational intensity of IDP, the comparisons between IDP and DDP are firstly made. Fig. 6 (a) illustrates the velocity profiles generated by different methods according to the set parameters, as listed in Table 7. In addition, Fig. 6 (a) also provides the control inputs comparison among four different methods. A 1500 m route with the default velocity limits is considered, and the target travel time is $200 \mathrm{~s}$. In Table 7, three DDP implementation cases are applied with three sets of grid numbers. According to Fig. 6 (a) and Table 7, the travel time becomes closer to the target value solved by DDP with the increment of numbers of the discrete control and state variables in each step. The improved performance incurred by the increased numbers of control and state variables, nevertheless, imposes huge computation burden which is reflected by the augmented calculation time. The travel time target can be precisely met by the IDP with less computation time, proving the superior performance of IDP in DBO, compared to that of DDP. Moreover, the velocity profiles generated by IDP with travel time constraint enable the motor to operate in higher efficiency region, thus contributing to less energy consumption in total.

Table 7 Numerical results by IDP and DDP

\begin{tabular}{|c|c|c|c|c|c|c|c|c|c|c|}
\hline Method & $n_{x}$ & $n_{u}$ & $\begin{array}{l}\Delta s \\
(\mathrm{~m})\end{array}$ & $S_{t}(\mathrm{~m})$ & $T_{\text {cal }}(\mathrm{s})$ & $\begin{array}{l}\text { Ini_X } \\
(\mathrm{m} / \mathrm{s})\end{array}$ & $\begin{array}{l}\text { Ter_X } \\
(\mathrm{m} / \mathrm{s})\end{array}$ & $\begin{array}{l}E_{-} \text {con } \\
(\mathrm{kWh})\end{array}$ & $\begin{array}{l}T_{-} t a r \\
\text { (s) }\end{array}$ & $\begin{array}{l}T_{\text {_tra }} \\
\text { (s) }\end{array}$ \\
\hline DP_case1 & 200 & 200 & 5 & 1500 & 232.9236 & 0 & 2.9922 & 0.3153 & 200 & 208.63 \\
\hline DP_case2 & 400 & 400 & 5 & 1500 & 686.3344 & 0 & 2.9934 & 0.3119 & 200 & 205.32 \\
\hline DP_case3 & 600 & 600 & 5 & 1500 & 863.7402 & 0 & 2.9973 & 0.3071 & 200 & 202.19 \\
\hline IDP & $55-85$ & $50-75$ & 5 & 1500 & 87.2659 & 0 & 2.9995 & 0.3016 & 200 & 200.21 \\
\hline
\end{tabular}

Note: $\Delta s$ is the simulation step, $S_{t}$ it the total travel time, $T_{c a l}$ is the algorithm calculation time, Ini_ $x$ and Ter_ $x$ are the initial speed and target speed, $E_{-}$con is the energy consumption on the route, $T_{-} \operatorname{tar}$ is the target travel time on the route, and $T \_t r a$ is the real travel time on the route. 

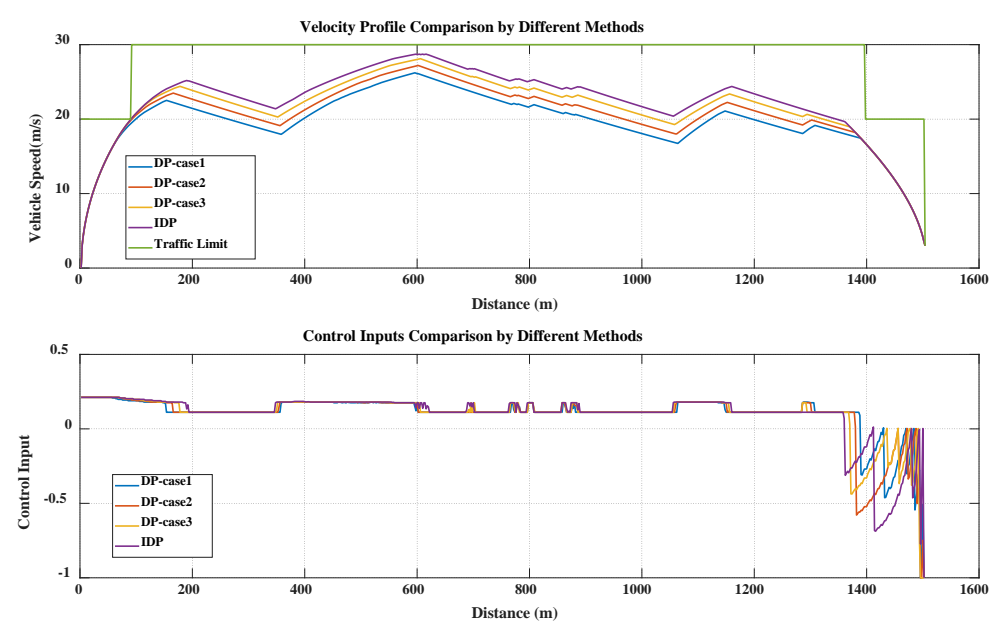

(a)
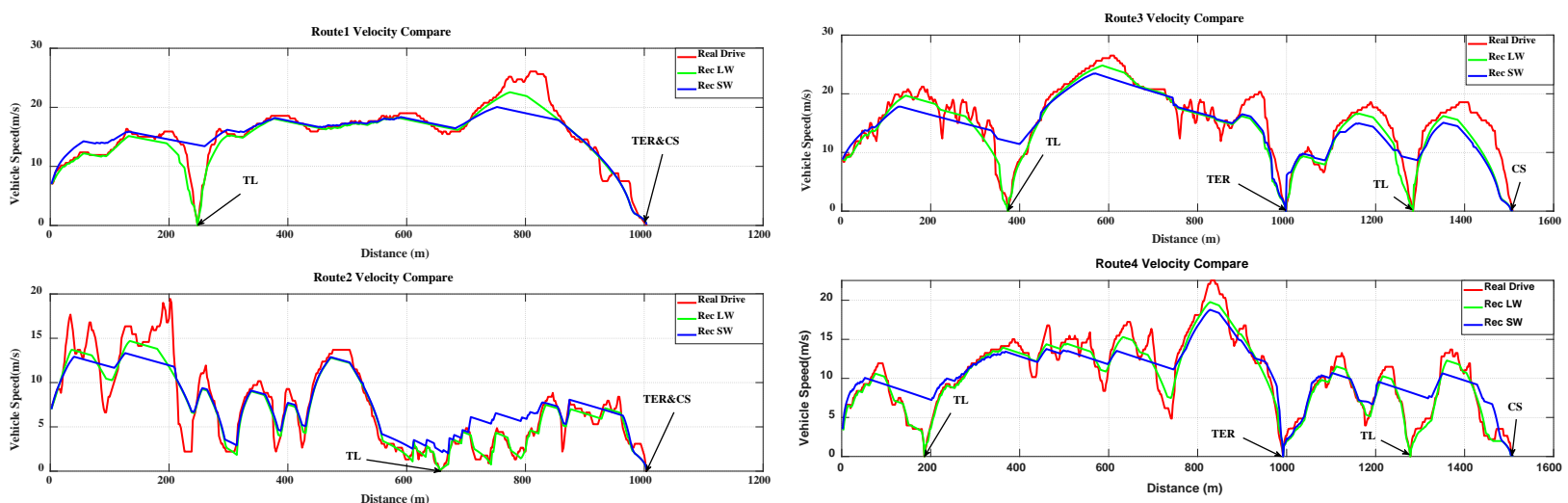

(b)

(c)
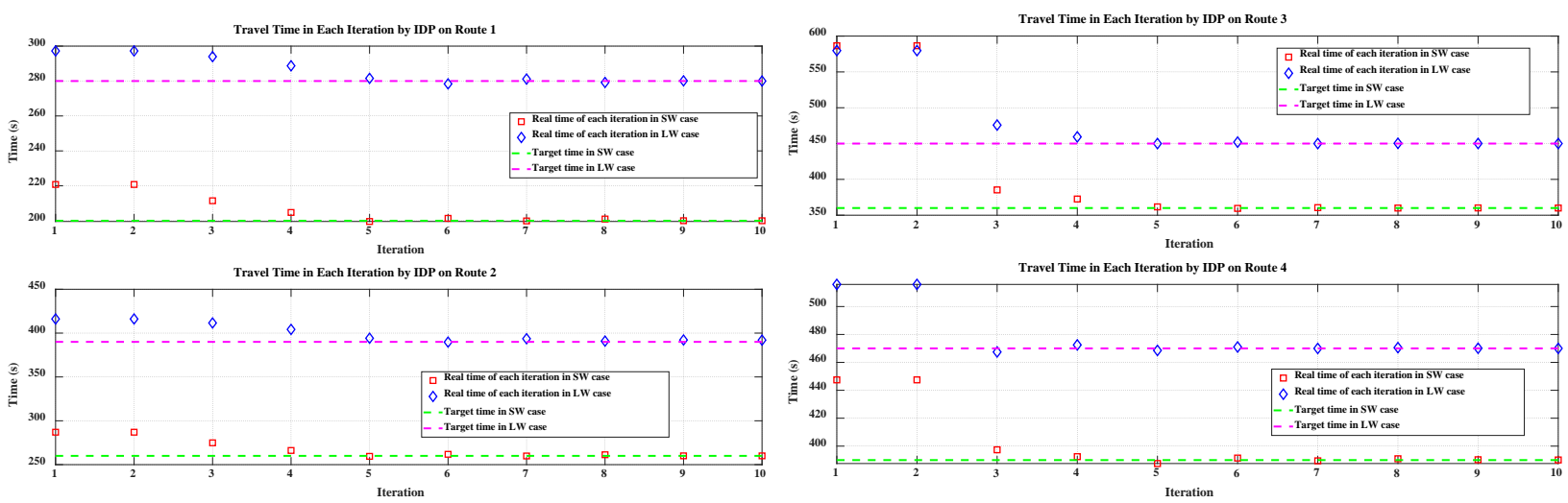

(d)

(e)

Fig. 6. Evaluation on reference velocity profile generation. (a) Comparison in velocity profiles and control inputs by IDP and DDP. (b) The optimized velocity profiles by IDP in route 1 and route 2. (c) The optimized velocity profiles by IDP in route 3 and route 4 . (d) Travel times in different iterations in route 1 and route 2. (e) Travel times in different iterations in route 3 and route 4.

In Table 8, the performance of existing methods in velocity profile optimization is further studied. As can be found, all preferred methods can generate optimal velocity profiles with the travel times of around $200 \mathrm{~s}$. Due to the specific iteration calculation in IDP, the optimal velocity profile by IDP achieves the travel time that approximates mostly to $200 \mathrm{~s}$. In addition, the calculation time by IDP is obviously shortened, compared with other methods. The computation time by IDP respectively occupies only $10.14 \%, 44.5 \%$, and $28.3 \%$ of 
the computing time by the DP, EDA, and QP, highlighting the preferable application potential in real-time practice.

Table 8 Compare results by different methods

\begin{tabular}{llllll}
\hline Method & $\Delta s(\mathrm{~m})$ & $S_{t}(\mathrm{~m})$ & $T_{\text {cal }}(\mathrm{s})$ & $T_{-}$tar $(\mathrm{s})$ & $T_{-}$tra $(\mathrm{s})$ \\
\hline DP_case 3 & 5 & 1500 & 863.7402 & 200 & 202.19 \\
EDA & 5 & 1500 & 197.1138 & 200 & 203.97 \\
QP & 5 & 1500 & 311.4963 & 200 & 203.34 \\
IDP & 5 & 1500 & 87.6259 & 200 & 200.21 \\
\hline
\end{tabular}

Note: $\Delta s$ is the simulation step, $S_{t}$ it the total travel time, $T_{c a l}$ is the algorithm calculation time, $T_{-}$tar is the target travel time on the route, and $T_{-}$tra is the real travel time on the route.

After proving the qualified capability of IDP in DBO, more in-depth investigation is performed to further assess the active role of IDP in velocity profile optimization. Fig. 6 (b) and (c) illustrate the velocity profiles derived from the real driving data or optimized by IDP on four routes in distance domain. Here, Rec LW refers to the recommended velocity profile under long charging pile available time, while Rec SW means the optimal velocity profile on the condition that the charging pile in the selected CS is available right now or the available time is less than the estimated travel time. TL means the traffic light on the route, and TER represents the travel destination. In the first two routes, the EV charging is required immediately without continuing to travel to destination. In the latter two cases, the EV charging is booked after the vehicle arrives at the destination. In the simulation, the traffic light switch timing sequence is assigned. The period between green and red light is $60 \mathrm{~s}$, and the period between red and green light is 45 s. According to Fig. 6 (b) and (c), the optimized velocity profiles in SW cases on four routes tend to guide the vehicle to avoid red light to reach the chosen CSs timely. By contrast, the optimized velocity profiles in LW cases on four routes incline to instruct the vehicle to stop at traffic lights to increase the total travel time, endeavoring to pilot the vehicle to arrive at the chosen CSs at the moment that the charging piles are just available. Fig. 6 (d) and (e) present the travel time adjustment in each iteration when implementing the IDP on the same four routes as before. By adaptively changing the weight ratio of cost functions in IDP, the travel time can be governed to approximate the target time. In Fig. 6 (d) and (e), the travel duration of the optimized velocity profiles tends to approach the target value after five iterations, proving the marvelous fast optimization search capability of IDP. Even though the travel time in each iteration is adjusted, the IDP algorithm can still guarantee the energy-efficient velocity profile in each iteration, which is also proved by the numerical results listed in Table 9.

Table 9 Numerical results by IDP on four routes

\begin{tabular}{|c|c|c|c|c|c|c|c|c|c|c|c|c|}
\hline Method & Case & $n_{x}$ & $n_{u}$ & $\begin{array}{l}\Delta s \\
(\mathrm{~m})\end{array}$ & $\begin{array}{l}S_{t} \\
(\mathrm{~m})\end{array}$ & $\begin{array}{c}T_{\text {cal }} \text { by } \\
\operatorname{MEC~(s)~}\end{array}$ & $\begin{array}{c}T_{\text {cal }} \text { by non- } \\
\text { MEC (s) }\end{array}$ & $\begin{array}{l}\text { Ini_x } \\
(\mathrm{m} / \mathrm{s})\end{array}$ & $\begin{array}{c}\text { Ter_x } \\
(\mathrm{m} / \mathrm{s})\end{array}$ & $\begin{array}{l}E_{-} \text {con } \\
(\mathrm{kWh})\end{array}$ & $\begin{array}{c}T_{-} \text {tar } \\
\text { (s) }\end{array}$ & $\begin{array}{c}T_{-} t r a \\
\text { (s) }\end{array}$ \\
\hline \multirow{3}{*}{$\begin{array}{l}\text { IDP } \\
\text { route1 }\end{array}$} & Rec LW & $45-85$ & $45-85$ & 5 & 1000 & 92.12 & 204.30 & 6.5 & 0.1711 & 0.0742 & 280 & 280 \\
\hline & Rec SW & $40-75$ & $45-75$ & 5 & 1000 & 72.79 & 180.97 & 6.5 & 0.1971 & 0.1088 & 200 & 200 \\
\hline & $\mathrm{RD}$ & - & - & - & 1000 & - & & 6.5 & 0 & 0.1239 & - & 254 \\
\hline \multirow{3}{*}{$\begin{array}{l}\text { IDP } \\
\text { route2 }\end{array}$} & Rec LW & $45-90$ & $40-85$ & 5 & 1000 & 93.12 & 206.65 & 3 & 0.1703 & 0.0809 & 390 & 390.06 \\
\hline & Rec SW & $45-80$ & $40-75$ & 5 & 1000 & 71.45 & 176.62 & 3 & 0.1717 & 0.0843 & 260 & 260.01 \\
\hline & $\mathrm{RD}$ & - & - & - & 1000 & - & & 3 & 0 & 1.1179 & - & 271 \\
\hline \multirow{3}{*}{$\begin{array}{l}\text { IDP } \\
\text { route3 }\end{array}$} & Rec LW & 50-95 & $45-85$ & 5 & 1500 & 101.46 & 234.45 & 8.5 & 0.1702 & 0.1984 & 450 & 450 \\
\hline & Rec SW & $50-90$ & $45-80$ & 5 & 1500 & 91.14 & 189.45 & 8.5 & 0.1711 & 0.2142 & 360 & 360.02 \\
\hline & $\mathrm{RD}$ & - & - & - & 1500 & - & & 8.5 & 0 & 0.2374 & - & 413 \\
\hline \multirow{2}{*}{$\begin{array}{l}\text { IDP } \\
\text { route4 }\end{array}$} & Rec LW & $55-95$ & $50-90$ & 5 & 1500 & 101.11 & 232.59 & 3 & 0.1711 & 0.1155 & 470 & 470.01 \\
\hline & Rec SW & $60-95$ & $55-90$ & 5 & 1500 & 89.13 & 186.42 & 3 & 0.1708 & 0.1504 & 390 & 390.09 \\
\hline
\end{tabular}


Table 9 lists the parameter sets of IDP and numerical results on four routes with MEC and non-MEC framework. Apparently, the computing time in different routes by the MEC based framework is less than those by the non-MEC based framework. The maximum time reduction by the MEC based framework can reach 59.78\%, highlighting the superior performance of the MEC based framework. It also shows that the CPU computation time is less than the total travel time with small numbers of discrete state and control variables, suggesting great potential in real-time implementation of IDP. In engineering practice, the optimal velocity profiles can be generated by IDP in MECUs before the vehicle enters the new segment. In Table 9, the prearranged travel time targets with different charging cases on four routes are all met. Compared with the real driving data, the generated velocity profiles achieve better energy economy in different charging cases. In Table 9, the total calculation times for the whole travel with the MEC based framework in route 1 and 2 ranges $72 \mathrm{~s}$ to $92 \mathrm{~s}$, and the total travel route is from $200 \mathrm{~s}$ to $390 \mathrm{~s}$. For each route segment, the average calculation time of MECU is around $36 \mathrm{~s}$ to $46 \mathrm{~s}$, and the average travel time is $100 \mathrm{~s}$ to $190 \mathrm{~s}$. In the proposed CMS, each MECU is requested to generate the optimal velocity profile for next route segment. The results in Table 9 prove that the optimal velocity profile can be prepared before the vehicle drives into next segment and validates that the proposed CMS characterizes reasonable capability in real-time application.

\subsubsection{Investigation of Optimal Diving Suggestion Generation}

The generated optimal velocity profiles are tracked by MPC in VCU to generate energy-efficiency suggestions for drivers or control commands sent to motor control units (MCU) to realize eco-driving. Fig. 7 shows the tracking results on two routes. It can be clearly observed that the MPC algorithm preferred in CMS can track the reference velocity profiles closely in most cases, guaranteeing that the optimal control policies generated in MECUs can be precisely realized by the vehicle powertrain. There also exist some minor tracking differences, which are caused by the errors existing in the nonlinear solving results. To be specific, the polynomial functions are employed to approximate the motor efficiency and the required total tractive torque, and definitely the estimation truthfulness on parameters in the polynomial functions can dominate the approximation accuracy conspicuously.

According to the simulation results, it can be summarized that the proposed CMS is qualified in both the charging booking and DBO. The employed nonlinear MPC effectively completes the reference velocity profile tracking, ensuring that the optimal control policies can be accurately executed by the vehicle powertrain. In short, the presented CMS enhances the reduction of charging waiting time and achieves the eco-charging simultaneously. 

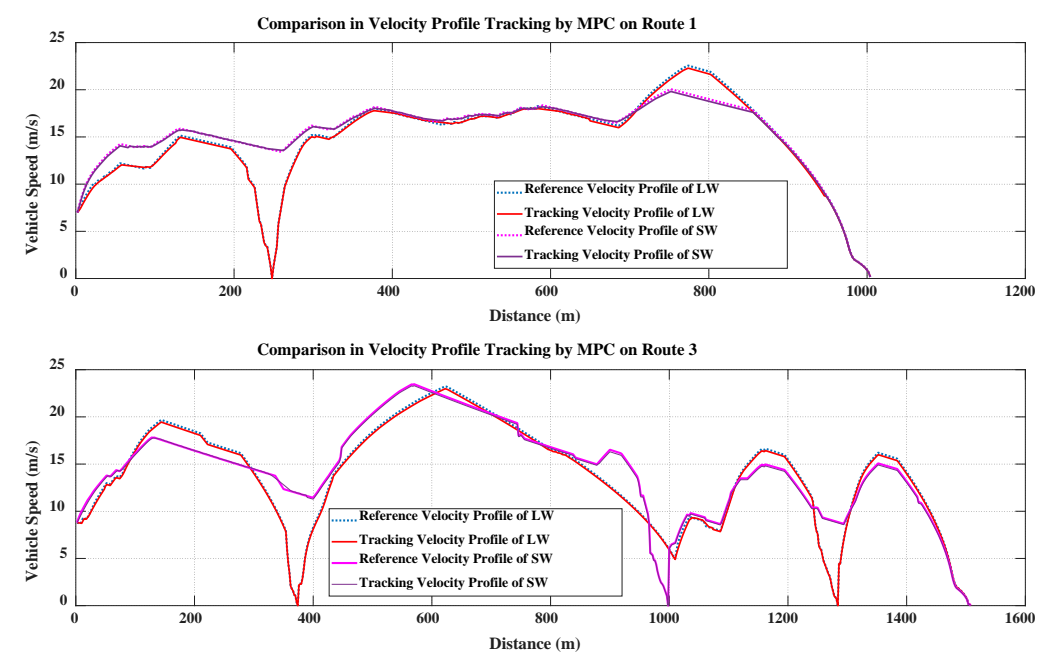

Fig. 7. Reference velocity profile tracking results.

\section{CONCLUSION}

This paper proposes a cooperative and efficient charging management strategy for EVs. Based on a service-oriented MEC framework, the novel CMS can realize charging booking and driving optimization chronologically. The carefully designed CBA in CMS can select the most suitable CS and plan the shortest route from current location to the chosen CS, thereby minimizing charging waiting time remarkably. The rarely considered DBO in CMS is accomplished by a two-step process by means of IDP and nonlinear MPC, endeavoring to reduce energy consumption during the trip to CS. The simulation analysis validates the superior performance of the proposed strategy compared with the existing algorithms.

In the future work, the CMS will be further investigated by extending the scope from individual EV to EV platoon. The positive and negative impact on grid incurred by CMSs will be further investigated, thus providing in-depth guide for further strategy development. Additionally, studies on DBO will be further conducted to realize personalized behavior optimization. Moreover, design of more adaptive and robust management algorithms that incorporate the vehicle's battery capacity variation incurred by operating temperature and aging effect will also be investigated in our future study.

\section{ACKNOWLEDGEMENT}

This work was supported in part by the National Natural Science Foundation of China (No. 61763021 and No. 51775063), in part by the National Key R\&D Program of China (No. 2018YFB0104900), and in part by the EU-funded Marie Skłodowska-Curie Individual Fellowships Project under Grant 845102-HOEMEVH2020-MSCA-IF-2018.

\section{REFERENCE}

[1] W. Zhou, L. Yang, Y. Cai, and T. Ying, "Dynamic programming for New Energy Vehicles based on their work modes part I: Electric Vehicles and Hybrid Electric Vehicles," Journal of Power Sources, vol. 406, pp. 151-166, 2018.

[2] W. Zhou, L. Yang, Y. Cai, and T. Ying, "Dynamic programming for new energy vehicles based on their work modes Part II: Fuel cell electric vehicles," Journal of Power Sources, vol. 407, pp. 92-104, 2018. 
[3] Y. Liu, J. Li, Z. Chen, D. Qin, and Y. Zhang, "Research on a multi-objective hierarchical prediction energy management strategy for range extended fuel cell vehicles," Journal of Power Sources, vol. 429, pp. 55-66, 2019/07/31/ 2019.

[4] Y. Zheng, M. Ouyang, X. Han, L. Lu, and J. Li, "Investigating the error sources of the online state of charge estimation methods for lithium-ion batteries in electric vehicles," Journal of Power Sources, vol. 377, pp. 161-188, 2018.

[5] L. Kouchachvili, W. Yaici, and E. Entchev, "Hybrid battery/supercapacitor energy storage system for the electric vehicles," Journal of Power Sources, vol. 374, pp. 237-248, 2018.

[6] J. Tian, Y. Wang, D. Yang, X. Zhang, and Z. Chen, "A real-time insulation detection method for battery packs used in electric vehicles," Journal of Power Sources, vol. 385, pp. 1-9, 2018.

[7] A. Burnham et al., "Enabling fast charging - Infrastructure and economic considerations," Journal of Power Sources, vol. 367, pp. 237-249, 2017.

[8] A. J. Cheng, B. Tarroja, B. Shaffer, and S. Samuelsen, "Comparing the emissions benefits of centralized vs. decentralized electric vehicle smart charging approaches: A case study of the year 2030 California electric grid," Journal of Power Sources, vol. 401, pp. 175-185, 2018.

[9] Y. Cao et al., "Toward Efficient, Scalable, and Coordinated On-the-Move EV Charging Management," IEEE Wireless Communications, vol. 24, no. 2, pp. 66-73, 2017.

[10] J. C. Mukherjee and A. Gupta, "A Review of Charge Scheduling of Electric Vehicles in Smart Grid," IEEE Systems Journal, vol. 9, no. 4, pp. 1541-1553, 2015.

[11] R. Hussain, D. Kim, M. Nogueira, J. Son, A. O. Tokuta, and H. Oh, "A New Privacy-Aware Mutual Authentication Mechanism for Charging-on-the-Move in Online Electric Vehicles," in mobile ad hoc and sensor networks, 2015, pp. 108115.

[12] Y. Cao, N. Wang, G. Kamel, and Y. Kim, "An Electric Vehicle Charging Management Scheme Based on Publish/Subscribe Communication Framework," IEEE Systems Journal, vol. 11, no. 3, pp. 1822-1835, 2017.

[13] B. Aluisio, A. Conserva, M. Dicorato, G. Forte, and M. Trovato, "Optimal operation planning of V2G-equipped Microgrid in the presence of EV aggregator," Electric Power Systems Research, vol. 152, pp. 295-305, 2017.

[14] S. Deilami, A. S. Masoum, P. S. Moses, and M. A. S. Masoum, "Real-Time Coordination of Plug-In Electric Vehicle Charging in Smart Grids to Minimize Power Losses and Improve Voltage Profile," IEEE Transactions on Smart Grid, vol. 2, no. 3, pp. 456-467, 2011.

[15] J. Lee, H. Kim, G. Park, and H. Jeon, "Genetic algorithm-based charging task scheduler for electric vehicles in smart transportation," in asian conference on intelligent information and database systems, 2012, pp. 208-217.

[16] T. M. Sweda and D. Klabjan, "Finding minimum-cost paths for electric vehicles," in ieee international electric vehicle conference, 2012, pp. 1-4.

[17] M. Alizadeh, H. Wai, A. Scaglione, A. J. Goldsmith, Y. Fan, and T. Javidi, "Optimized path planning for electric vehicle routing and charging," in allerton conference on communication, control, and computing, 2014, pp. 25-32.

[18] C. Wang et al., "Cellular architecture and key technologies for 5G wireless communication networks," IEEE Communications Magazine, vol. 52, no. 2, pp. 122-130, 2014.

[19] S. J. Russell and P. Norvig, "Artificial intelligence: a modern approach. Malaysia," ed: Pearson Education Limited, 2016.

[20] J. C. White, N. C. Coops, M. A. Wulder, M. Vastaranta, T. Hilker, and P. Tompalski, "Remote Sensing Technologies for Enhancing Forest Inventories: A Review," Canadian Journal of Remote Sensing, vol. 42, no. 5, pp. 619-641, 2016.

[21] Y. Cao, T. Wang, O. Kaiwartya, G. Min, N. Ahmad, and A. H. Abdullah, "An EV Charging Management System Concerning Drivers’ Trip Duration and Mobility Uncertainty," systems man and cybernetics, vol. 48, no. 4, pp. 596-607, 2018.

[22] E. Kural, S. Jones, A. F. Parrilla, and A. Grauers, "Traffic light assistant system for optimized energy consumption in an electric vehicle," in international conference on connected vehicles and expo, 2014, pp. 604-611.

[23] S. Bae, Y. Kim, J. Guanetti, F. Borrelli, and S. J. Moura, "Design and Implementation of Ecological Adaptive Cruise Control for Autonomous Driving with Communication to Traffic Lights," arXiv: Systems and Control, 2018.

[24] Q. Cheng, L. Nouveliere, and O. Orfila, "A new eco-driving assistance system for a light vehicle: Energy management and speed optimization," in ieee intelligent vehicles symposium, 2013, pp. 1434-1439.

[25] F. Mensing, E. Bideaux, R. Trigui, and H. Tattegrain, "Trajectory optimization for eco-driving taking into account traffic constraints," Transportation Research Part D-transport and Environment, vol. 18, pp. 55-61, 2013.

[26] L. Guo, H. Chen, Q. Liu, and B. Gao, "A Computationally Efficient and Hierarchical Control Strategy for Velocity Optimization of On-Road Vehicles," systems man and cybernetics, vol. 49, no. 1, pp. 31-41, 2019.

[27] H. Lim, C. C. Mi, and W. Su, "A Distance-Based Two-Stage Ecological Driving System Using an Estimation of Distribution Algorithm and Model Predictive Control," IEEE Transactions on Vehicular Technology, vol. 66, no. 8, pp. 6663-6675, 2017.

[28] G. Li and D. Gorges, "Ecological Adaptive Cruise Control and Energy Management Strategy for Hybrid Electric Vehicles Based on Heuristic Dynamic Programming," IEEE Transactions on Intelligent Transportation Systems, pp. 1-10, 2018.

[29] H. Lim, W. Su, and C. C. Mi, "Distance-Based Ecological Driving Scheme Using a Two-Stage Hierarchy for Long-Term Optimization and Short-Term Adaptation," IEEE Transactions on Vehicular Technology, vol. 66, no. 3, pp. 1940-1949, 2017.

[30] V. Doan, H. Fujimoto, T. Koseki, T. Yasuda, H. Kishi, and T. Fujita, "Iterative Dynamic Programming for Optimal Control Problem with Isoperimetric Constraint and Its Application to Optimal Eco-driving Control of Electric Vehicle," IEEJ journal of industry applications, vol. 7, no. 1, pp. 80-92, 2018.

[31] C. Montzka, H. Moradkhani, L. Weihermuller, H. J. H. Franssen, M. J. Canty, and H. Vereecken, "Hydraulic parameter estimation by remotely-sensed top soil moisture observations with the particle filter," Journal of Hydrology, vol. 399, no. 3, pp. 410-421, 2011.

[32] Chen, Zheng, Yuhong Fu, and Chunting Chris Mi. "State of charge estimation of lithium-ion batteries in electric drive vehicles using extended Kalman filtering." IEEE Transactions on Vehicular Technology 62.3 (2012): 1020-1030.

[33] M. N. Tehrani, M. Uysal, and H. Yanikomeroglu, "Device-to-device communication in 5G cellular networks: challenges, solutions, and future directions," IEEE Communications Magazine, vol. 52, no. 5, pp. 86-92, 2014. 
[34] Q. Kang, J. Wang, M. Zhou, and A. C. Ammari, "Centralized Charging Strategy and Scheduling Algorithm for Electric Vehicles Under a Battery Swapping Scenario," IEEE Transactions on Intelligent Transportation Systems, vol. 17, no. 3, pp. 659-669, 2016.

[35] S. Zhang, W. Dou, Y. Zhang, W. Hao, Z. Chen, and Y. Liu, "A Vehicle-Environment Cooperative Control Based Velocity Profile Prediction Method and Case Study in Energy Management of Plug-in Hybrid Electric Vehicles," IEEE Access, vol. 7, pp. 75965-75975, 2019.

[36] X. Gao, L. Dai, and A. M. Sayeed, "Low RF-complexity technologies to enable millimeter-wave MIMO with large antenna array for 5G wireless communications,” IEEE Commun. Mag., vol. 56, no. 4, pp. 211-217, Apr. 2018.

[37] N. T. Milas and E. C. Tatakis, "Charging Station Selection through the Analytic Hierarchy Process enabled by OPC-UA for Vehicle-to-Grid Communications," 2019 21st European Conference on Power Electronics and Applications (EPE '19 ECCE Europe), Genova, Italy, 2019, pp. P.1-P.10.

[38] Jahangir, H., Tayarani, H., Ahmadian, A., Golkar, M. A., Miret, J., Tayarani, M., \& Gao, H. O.. "Charging demand of Plugin Electric Vehicles: Forecasting travel behavior based on a novel Rough Artificial Neural Network approach." Journal of cleaner production 229 (2019): 1029-1044.

[39] P. Sudhakara and V. Ganapathy, "Trajectory Planning of a Mobile Robot using Enhanced A-Star Algorithm," Indian journal of science and technology, vol. 9, no. 41, 2016.

[40] Y. Zhang et al., "Optimal energy management strategy for parallel plug-in hybrid electric vehicle based on driving behavior analysis and real time traffic information prediction," Mechatronics, vol. 46, pp. 177-192, 2017.

[41] O. C. Map, "The Open Charge Map API," ed, 2016.

[42] M. Haklay and P. Weber, "OpenStreetMap: User-Generated Street Maps," IEEE Pervasive Computing, vol. 7, no. 4, pp. 1218, 2008. 